\title{
Depopulation of dense a-synuclein aggregates is associated with rescue of dopamine neuron dysfunction and death in a new Parkinson's disease model
}

\author{
Michal Wegrzynowicz ${ }^{1}$ Dana Bar-On ${ }^{2,3}$. Laura Calo' ${ }^{1}$ - Oleg Anichtchik ${ }^{1}$ Mariangela lovino ${ }^{1}$. Jing Xia ${ }^{4}$. \\ Sergey Ryazanov ${ }^{5,6}$. Andrei Leonov ${ }^{5,6} \cdot$ Armin Giese $^{7}$. Jeffrey W. Dalley ${ }^{4,8} \cdot$ Christian Griesinger $^{5,6,9} \cdot$ Uri Ashery $^{2,3}$. \\ Maria Grazia Spillantini ${ }^{1}$ (1)
}

Received: 14 January 2019 / Revised: 20 April 2019 / Accepted: 30 April 2019 / Published online: 31 May 2019

(c) The Author(s) 2019

\begin{abstract}
Parkinson's disease (PD) is characterized by the presence of $\alpha$-synuclein aggregates known as Lewy bodies and Lewy neurites, whose formation is linked to disease development. The causal relation between $\alpha$-synuclein aggregates and PD is not well understood. We generated a new transgenic mouse line (MI2) expressing human, aggregation-prone truncated 1-120 $\alpha$-synuclein under the control of the tyrosine hydroxylase promoter. MI2 mice exhibit progressive aggregation of $\alpha$-synuclein in dopaminergic neurons of the substantia nigra pars compacta and their striatal terminals. This is associated with a progressive reduction of striatal dopamine release, reduced striatal innervation and significant nigral dopaminergic nerve cell death starting from 6 and 12 months of age, respectively. In the MI2 mice, alterations in gait impairment can be detected by the DigiGait test from 9 months of age, while gross motor deficit was detected by rotarod test at 20 months of age when $50 \%$ of dopaminergic neurons in the substantia nigra pars compacta are lost. These changes were associated with an increase in the number and density of 20-500 $\mathrm{nm} \alpha$-synuclein species as shown by $d$ STORM. Treatment with the oligomer modulator anle 138b, from 9 to 12 months of age, restored striatal dopamine release, prevented dopaminergic cell death and gait impairment. These effects were associated with a reduction of the inner density of large $\alpha$-synuclein aggregates and an increase in dispersed small $\alpha$-synuclein species as revealed by $d$ STORM. The MI2 mouse model recapitulates the progressive dopaminergic deficit observed in PD, showing that early synaptic dysfunction is associated to fine behavioral motor alterations, precedes dopaminergic axonal loss and neuronal death that become associated with a more consistent motor deficit upon reaching a certain threshold. Our data also provide new mechanistic insight for the effect of anle138b's function in vivo supporting that targeting $\alpha$-synuclein aggregation is a promising therapeutic approach for PD.
\end{abstract}

Keywords Alpha-synuclein $\cdot$ Mouse model $\cdot$ Substantia nigra $\cdot$ Striatum $\cdot$ Anle138b $\cdot d$ STORM $\cdot$ Parkinson's disease

Electronic supplementary material The online version of this article (https://doi.org/10.1007/s00401-019-02023-x) contains supplementary material, which is available to authorized users.

Maria Grazia Spillantini

mgs11@cam.ac.uk

1 Department of Clinical Neurosciences, University of Cambridge, The Clifford Allbutt Building, Cambridge CB2 0AH, UK

2 School of Neurobiology, Biochemistry and Biophysics, George S. Wise Faculty of Life Sciences, Tel Aviv, Israel

3 Sagol School of Neuroscience, Tel Aviv University, 69978 Tel Aviv, Israel

4 Department of Psychology, University of Cambridge, Cambridge CB2 3EB, UK
5 Max Planck Institute for Biophysical Chemistry, 37077 Göttingen, Germany

6 DFG Research Centre Nanoscale Microscopy and Molecular Physiology of the Brain, 37070 Göttingen, Germany

7 Center for Neuropathology and Prion Research, Ludwig Maximilians University Munich, 81377 Munich, Germany

8 Department of Psychiatry, University of Cambridge, Cambridge CB2 2SZ, UK

9 Cluster of Excellence "Multiscale Bioimaging: from Molecular Machines to Networks of Excitable Cells" (MBExC), University of Göttingen, Göttingen, Germany 


\section{Introduction}

Parkinson's disease (PD) and other $\alpha$-synucleinopathies are characterized by aggregation of $\alpha$-synuclein $(\alpha \operatorname{Syn})$ in Lewy bodies (LBs), Lewy neurites (LNs) and glial cytoplasmic inclusions [17, 32, 33]. It is now widely accepted that the process of $\alpha$ Syn aggregation in PD is directly involved in the pathogenesis and progression of the disease, and motor symptoms are mainly related to the dysfunction of the nigrostriatal dopamine (DA) system. Indeed, an impairment of DA neurotransmission in the striatum and loss of DA neurons in substantia nigra pars compacta $(\mathrm{SNpc})$ are observed at the time of $\mathrm{PD}$ diagnosis [16]. To date, there is no treatment that affects the mechanism of the disease, and existing therapies are symptomatic.

Availability of an animal model of PD that reproduces progressive DA dysfunction and DA neuronal death with progressive $\alpha$ Syn aggregation in the nigrostriatal system is crucial for understanding disease mechanisms and the testing of novel therapies for treating movement deficit. In addition, until recently it was difficult to study quantitatively the early steps of $\alpha$ Syn aggregation and the effects of aggregate inhibitors in a living system, mainly due to the limitations in the diffraction of conventional microscopy. Today, with the use of super resolution microscopy like STORM, $d$ STORM, STED and aptamer DNA PAINT [30] and advanced analysis algorithms [3], it is possible to identify changes in $\alpha$ Syn aggregation at the single molecule level also in in vivo systems and understand the mechanistic nature of aggregate inhibitors.

Here, we report a new transgenic mouse model (MI2 mice) expressing human 1-120 truncated $\alpha$ Syn that develops a progressive phenotype including $\alpha$ Syn aggregation, loss of striatal DA, reduction in induced striatal DA release, DA neuron death in SNpc, loss of nigrostriatal dopaminergic innervation, and motor deficits. We show that progressive DA dysfunction is associated with increased formation of synaptic striatal $\alpha$ Syn aggregates. Moreover, we show that the oligomer modulator, anle $138 \mathrm{~b}$, previously shown to be effective in other models of protein aggregation including $\alpha$ Syn $[15,23,36,37]$, restores striatal DA release and prevents DA cell loss and motor behavior even when administration is started after the onset of DA dysfunction. For the first time and using $d$ STORM and immunoblotting, we show that in vivo, in mouse brain, anle $138 \mathrm{~b}$ rescue of the DA deficit is associated with reduction of the density of dense $\alpha$ Syn aggregates and an increase in dispersed monomeric and small assemblies of $1-120 \mathrm{~h} \alpha \mathrm{Syn}$.

\section{Materials and methods}

\section{Study design}

This study aimed to generate and characterize a novel transgenic mouse model of PD that would enhance our understanding of the relationship between progressive $\alpha$ Syn aggregation and mechanisms of disease and allow testing prospective therapies for $\alpha$-synucleinopathies. The homozygous line expressing 1-120 h $\alpha$ Syn under the TH promoter, in a null endogenous $\alpha$ Syn background was generated and used for the experiments. The $\alpha$ Syn-null C57B1/6S strain was used as a control line. C57Bl/6J mice, expressing endogenous mouse $\alpha$ Syn were also included in the experiments where transgenic protein expression was compared to physiological mouse $\alpha$ Syn. Moreover, DA characteristics in MI2 mice were compared with both $\mathrm{C} 57 \mathrm{~B} 1 / 6 \mathrm{~S}$ and $\mathrm{C} 57 \mathrm{~B} 1 / 6 \mathrm{~J}$ mice to confirm that differences between $\mathrm{C} 57 \mathrm{~B} 1 / 6 \mathrm{~S}$ and MI2 mouse lines were due to the expression of the transgene rather than the absence of $\alpha \mathrm{Syn}$ in the $\mathrm{C} 57 \mathrm{Bl} / 6 \mathrm{~S}$ animals.

MI2 and control mice were obtained from separate lines, therefore their genotypes were known at the time of experimental design. However, for behavioral experiments and anle138b-treatment mice were assigned randomly to the experimental groups. For each experiment, mouse numbers and statistical tests are described in the figure legends and in Online Resource. Analysis of dopaminergic impairment, namely measurements of DA in striatal homogenates and microdialysates, stereological counting of nigral cells and striatal innervation were performed by researchers blinded to experimental conditions.

\section{Generation of transgenic mice}

The MI2 mouse line was generated using the same procedures as described by Tofaris et al. for $\alpha$-Syn 120 mice [35]. Briefly, a transgene construct containing human truncated 1-120 $\alpha$ Syn, subcloned downstream of the rat TH promoter (Fig. 1a) was injected into $\alpha$ Syn-null C57Bl/6OlaHsd (C57B1/6S) mouse [31] pronuclei. The presence of the transgene was detected in the founders and progeny by PCR. Founders were then bred with $\alpha$ Syn-null C57Bl/6S mice and mouse $\alpha$ Syn-negative/1-120 h $\alpha$ Syn-positive progeny were crossed to homozygosity.

\section{Western blotting}

Animals were sacrificed by cervical dislocation, brains were snap frozen on dry ice and kept at $-80{ }^{\circ} \mathrm{C}$. Brain regions were isolated, homogenized in ice-cold PBST $[1 \times$ PBS (Gibco), 0.3\% Triton X-100 (Sigma), protease inhibitor 
cocktail (Roche)] and centrifuged at $4{ }^{\circ} \mathrm{C}$, at $14,000 \times g$, for $15 \mathrm{~min}$. Supernatant protein content was measured using a BCA protein assay kit (Novagen) and protein concentration normalized using PBST.

To analyze the solubility of $1-120-\mathrm{h} \alpha$ Syn species, sequential extraction of protein was performed using buffers of increasing strength. Following dissection, tissue was homogenized in TBS, incubated on ice for $15 \mathrm{~min}$ and centrifuged at $120,000 \times g$ for $30 \mathrm{~min}$, at $4{ }^{\circ} \mathrm{C}$. The supernatant was collected for western blotting analysis, and the pellet was washed three times with TBS, resuspended in TBS + $1 \%$ Triton-X100, incubated on ice for $15 \mathrm{~min}$ and centrifuged at $120,000 \times g$ for $30 \mathrm{~min}$ at $4{ }^{\circ} \mathrm{C}$. These steps were then repeated using RIPA buffer (50 mM Tris, $\mathrm{pH} 8.0,150 \mathrm{mM}$ $\mathrm{NaCl}, 0.1 \%$ SDS, $0.05 \%$ sodium deoxycholate, $1 \% \mathrm{NP}-40$ ), and $8 \mathrm{M}$ urea (Sigma) with 5\% SDS (Sigma), with the exception that urea/SDS step was performed at room temperature rather than $4{ }^{\circ} \mathrm{C}$. All the solutions used in this procedure were supplemented with $1 \times$ protease inhibitor cocktail.

Following 5 min at $95{ }^{\circ} \mathrm{C}$ in $1 \times$ Laemmli buffer, samples were separated by SDS-PAGE, blotted onto nitrocellulose membranes (Bio-Rad) and proteins were crosslinked to the membrane with $4 \%$ paraformaldehyde (PFA) for $30 \mathrm{~min}$. Non-specific background was blocked with $5 \%$ milk in TBST before overnight incubation of the membrane at $4{ }^{\circ} \mathrm{C}$ with primary antibodies (mouse anti- $\alpha$ Syn (Syn 1), BD Biosciences, 1:500; rabbit anti-TH, Abcam, 1:1000; rabbit anti- $\beta$-actin, Abcam, 1:10,000) in 5\% milk. Membranes were then incubated with peroxidase-conjugated secondary antibodies (GE Healthcare or DAKO, 1:5000) and the blots imaged using a Chemi Doc MP imager (Bio-Rad), using West Dura Extended Duration Chemiluminescent Substrate (Thermo Fisher Scientific). Blots were analyzed using Image Lab 5.1 (Bio-Rad Laboratories).

Bands corresponding to mouse full length monomeric $(\sim 17 \mathrm{kDa})$ and $1-120$ truncated human $\alpha \operatorname{Syn}(\sim 14 \mathrm{kDa})$ were observed in C57Bl/6J and MI2 mice, respectively. No $\alpha$ Syn was present in C57Bl/6S (Fig. 1c). Non-specific bands recognized by the Syn 1 antibody were previously reported (Fig. 2c) [29].

\section{Immunohistochemistry}

Mice were anesthetized by an intraperitoneal administration of pentobarbital (Merial Animal Health) and transcardially perfused with ice-cold PBS, followed by $4 \%$ PFA in PBS. Brains were post-fixed overnight in 4\% PFA, then transferred to $30 \%$ sucrose in PBS with $0.1 \% \mathrm{NaN}_{3}$ (Sigma) and stored at $4{ }^{\circ} \mathrm{C}$. Brains were frozen and $30 \mu \mathrm{m}$ sections cut using a freezing microtome (Bright). Endogenous peroxidase activity was inhibited by treatment with $3 \% \mathrm{H}_{2} \mathrm{O}_{2}$ containing 20\% methanol in PBST. Sections non-specific background was blocked with 5\% normal horse serum (NHS,
Vector) in PBST. Antigen retrieval was performed before Syn1 staining, using $10 \mathrm{mM}$ sodium citrate buffer $\mathrm{pH} 8.5$ at $80{ }^{\circ} \mathrm{C}$ for $30 \mathrm{~min}$. Free floating sections were incubated overnight at RT with primary antibodies diluted in PBST (Syn 1, 1:500; rabbit or chicken anti-TH, Abcam, 1:1000; mouse anti-NeuN, Millipore, 1:1000, rabbit anti-VAMP2, Abcam, 1:250; rabbit anti-ubiquitin (Ubi-1), Millipore, 1:500) and then with biotinylated (Vector, 1:2000) or Alexa Fluor-conjugated (Thermo Fisher Scientific, 1:500) secondary antibodies diluted in PBST containing 5\% NHS. Peroxidase-based staining was developed using Vectastain Elite ABC HRP Kit (Vector) and DAB Peroxidase Substrate Kit (Vector). Sections were mounted on microscope slides, dehydrated, cleared in xylene and coverslipped using DPX Mountant (Sigma). In some experiments, sections were counterstained with $0.1 \%$ cresyl violet (Sigma). For immunofluorescence nuclei were counterstained with DAPI $(0.5 \mu \mathrm{g} / \mathrm{ml}$; Roche) and sections coverslipped using FluorSave mounting medium (Calbiochem).

To determine the presence of filamentous $\alpha$ Syn aggregates, brain sections from MI2 and control mice were incubated with either Thioflavin T (TfT, Sigma; $0.2 \mu \mathrm{M}$ in deionized water, for $10 \mathrm{~min}$ ) or pentameric formyl thiophene acetic acid (pFTAA, kind gift from P. Nilsson; $0.5 \mu \mathrm{M}$ in PBST, for $45 \mathrm{~min}$ ). $\alpha$ Syn staining with Syn1 antibody, revealed by $647 \mathrm{~nm}$ Alexa Fluor-conjugated secondary antibody, was performed before TfT or pFTAA to determine the specificity of the staining. Sections were washed extensively with PBST before incubation with DAPI. To further investigate the solubility of the $\alpha$ Syn aggregates serial sections were treated with proteinase PK (PK; Sigma) prior to $\alpha$ Syn immunohistochemistry to digest soluble $\alpha$ SYN. Prior to the non-specific background blocking step, the slices were incubated with PK $\left(20 \mu \mathrm{g} / \mathrm{ml}, 10 \mathrm{~min}, 37^{\circ} \mathrm{C}\right.$, at RT), and washed twice with PBST supplemented with $5 \mathrm{mM}$ phenylmethylsulfonyl fluoride (PMSF; Sigma) to inhibit residual PK activity. For these experiments immunostaining to detect $\alpha$ Syn was performed with Syn 1 antibody as described above. DAB-stained sections were analyzed using Olympus BX 53 light microscope and fluorescent sections using a Leica DMI 4000B epifluorescent microscope.

For $d$ STORM, $30 \mu \mathrm{m}$ free floating striatal slices were stained using a similar protocol, but PBST containing $0.25 \%$ Tween 20 was used and the non-specific background blocking solution contained $5 \%$ goat serum and $1 \%$ bovine serum albumin. Sections were stained with Syn204 mouse antihuman $\alpha$ Syn antibody (Abcam, 1:250) and Alexa Fluor 647 secondary anti-mouse antibody (Thermo Fisher Scientific, 1:500). In some experiments striatal sections were costained with rabbit anti-synaptobrevin 2 (VAMP2) antibody (Synaptic Systems, 1:500), followed by Cy3B anti-rabbit secondary antibody $(1: 250)$ to confirm synaptic localization of $1-120 \mathrm{~h} \alpha$ Syn by $d$ STORM. 
a

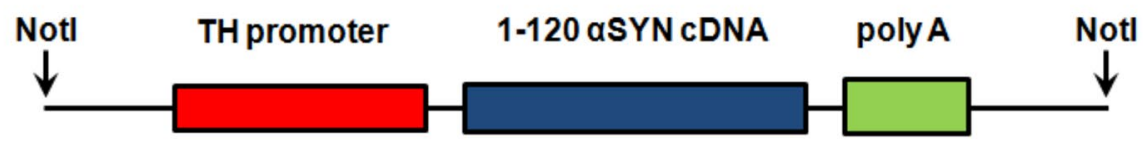

b
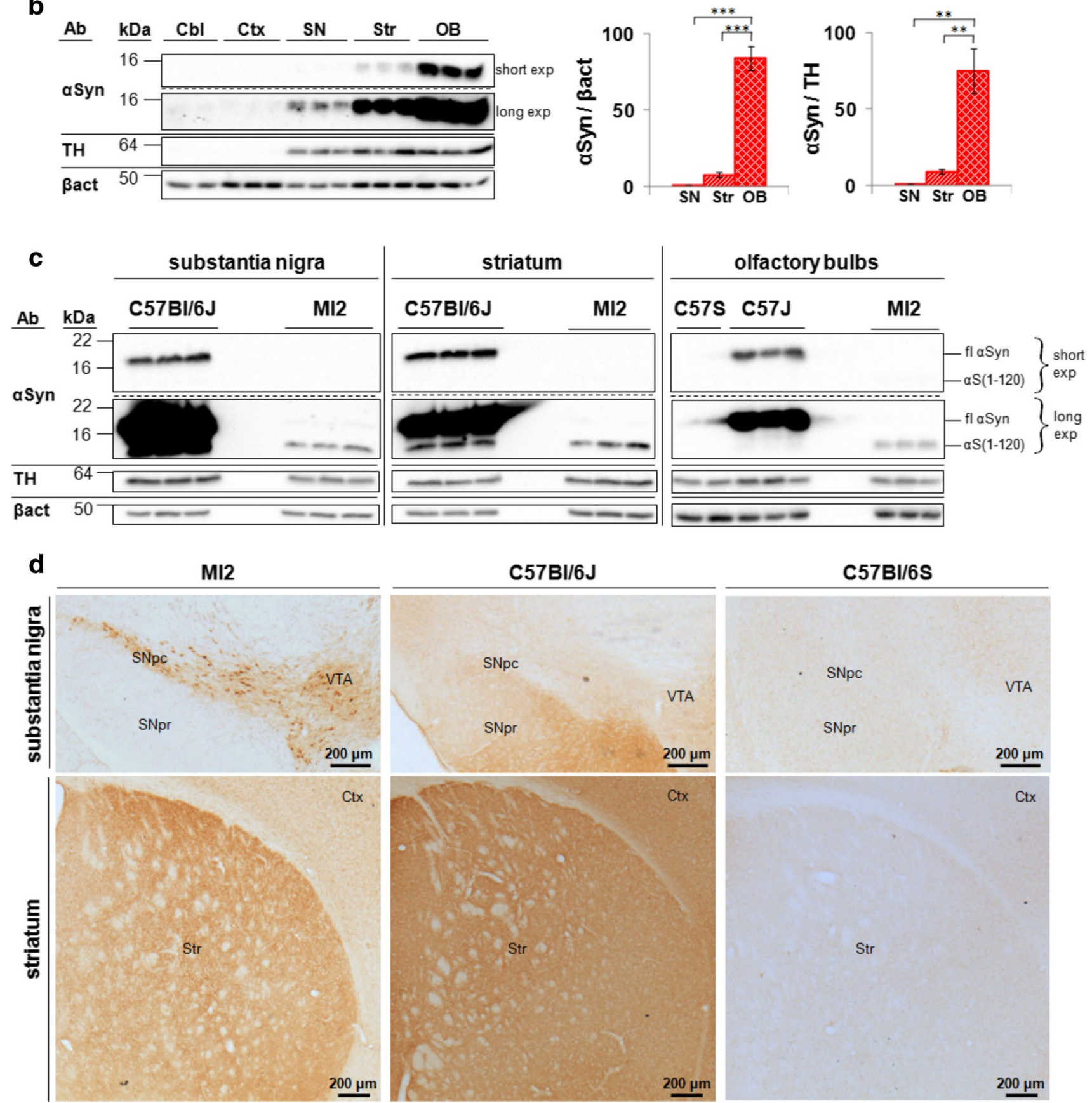

\section{dSTORM}

$d$ STORM images were taken using the Vutara microscope as described in [3], at power of $40 \% 647$ and $2 \% 405$ laser, and 3000 frames were taken for each movie. Localizations were gathered following the movie with a ratio of 10 of background to noise. All movies were then post analyzed using a denoise level of 0.5 .

Cluster analysis, mean diameter cluster analysis and the number of localizations per clusters were determined using the density based algorithm (dbscan) followed by $2 \mathrm{D}$ principal component analysis and outliers detection as described in [3] (see Supplementary Fig. S4, Online Resource 1 for more 
४Fig. 1 Expression of transgenic 1-120 hoSyn in MI2 mice. a 1-120 hoSyn transgene construct used for generating the MI2 mice. b Immunoblots showing $\alpha \mathrm{Syn}$ in lysates from cerebellum $(\mathrm{Cbl})$, cortex (Ctx), substantia nigra (SN), striatum (Str) and olfactory bulbs (OB) of 1.5 month-old mice following short (short exp) and long (long exp) exposure times. Quantification was performed for SN, Str and $\mathrm{OB}$, but not for $\mathrm{Cbl}$ and $\mathrm{Ctx}$, where the signal was negligible. Data are expressed as fold difference compared to SN (mean \pm SEM, $n=3$ mice, one-way ANOVA with Bonferroni correction $(* * p<0.01$, $* * * p<0.001$ ) (detailed statistics in Online Resource). c Immunoblots comparing expression levels between control and MI2 mouse lines. Expression of $1-120 \mathrm{~h} \alpha \mathrm{Syn}(\alpha \mathrm{S}(1-120))$ in MI2 mice is much lower than that of endogenous full-length $\alpha$ Syn (fl) in WT C57Bl/6J in the OB. Interestingly some truncated $\alpha$ Syn with a similar size to the transgenic 1-120 hoSyn can be seen in protein extracts of SN and Str of WT C57B1/6J mice. d Immunohistochemistry of brain sections of 1.5 month-old MI2 mice detected with the Syn1 antibody shows 1-120 hoSyn protein in neuronal cell bodies and processes in SNpc and ventral tegmental area (VTA) and in neuropil in striatum (see also Supplementary Fig. S1a, Online Resource 1). In C57B1/6J mice, endogenous $\alpha \mathrm{Syn}$, is also found in $\mathrm{SN}$ pars reticulata (SNpr) and $\mathrm{Ctx}$ besides SNpc, VTA and Str. The specificity of Syn1 antibody for $\alpha$ Syn was confirmed by the absence of staining in C57B1/6S mice that lack endogenous $\alpha$ Syn

details). For anle $138 \mathrm{~b}$ vs placebo treatment analysis, clusters in both conditions were additionally filtered according to inner density threshold determined according to the placebo condition per imaging day to avoid large-homogenous distributions that could form artifact clustering. Mean density of cluster was calculated for the placebo condition on the individual day of measurement and clusters with less than $10 \%$ of this density were excluded from analysis in both placebo and anle $138 \mathrm{~b}$ treatment conditions measured on the same day. The localizations that were accounted for very low-density clusters were in both cases added to the free (unclustered) populations of the proteins.

Additional information on analysis of $d$ STORM data are given in Online Resource.

\section{Dopamine and DOPAC measurements}

Bilateral striata were isolated from frozen brains, weighed and homogenized in $0.2 \mathrm{M}$ perchloric acid. Homogenates were centrifuged at $6000 \times g$ for $20 \mathrm{~min}$ at $4{ }^{\circ} \mathrm{C}$. The supernatants were resolved by high-performance liquid chromatography with a Hypersil BDS C18 reversed phase column (3 $\mu \mathrm{m}$ particle size, $130 \AA$ pore size, $100 \times 4.6 \mathrm{~mm}$; Phenomenex) at a flow rate of $1 \mathrm{ml} / \mathrm{min}$ as previously reported [12]. The mobile phase comprised of citric acid $(31.9 \mathrm{~g} / \mathrm{l})$, sodium acetate (2 $\mathrm{g} / \mathrm{l})$, octanesulfonic acid $(460 \mathrm{mg} / \mathrm{l})$, EDTA (30 mg/l) and methanol (15\%), pH 3.6. DA, and its metabolite, 3,4-dihydroxyphenylacetic acid (DOPAC) were detected by redox oxidation using an ESA 5014 analytical cell and ESA Coulochem II electrochemical detector (Thermo Fisher Scientific) with reducing (E1) and oxidizing (E2) electrodes set at $-200 \mathrm{mV}$ and $+250 \mathrm{mV}$, respectively.
The chromatograms were analyzed using the Chromeleon Chromatography Data System (V 6.2; Dionex). DA and DOPAC levels were normalized to the initial wet tissue weight and expressed as pmol/mg.

\section{In vivo microdialysis}

Animals were anesthetized with Caprieve and isoflurane and placed in a stereotaxic frame under general anaesthesia. A craniotomy was drilled in the skull according to the following coordinates relative to bregma: +0.8 anteroposterior, +2.1 lateral [28]. The guide cannula CMA7 (CMA Microdialysis) was implanted in the brain, so that its tip reached the callosostriatal border, $-2.3 \mathrm{~mm}$ dorsoventral relative to the skull surface [28], and was secured to the skull using glass ionomer luting cement FujiCEM 2 (GC Corporation) and two anchor screws CMA7431021 (CMA Microdialysis). Microdialysis was performed $24 \mathrm{~h}$ following the surgery when the mice had recovered. A microdialysis probe CMA7 $(0.24 \mathrm{~mm} \times 2 \mathrm{~mm}$ membrane, 6 kDa cut-off; CMA Microdialysis) was inserted through the guide cannula, and freely-moving mice were infused with artificial cerebro-spinal fluid (ACSF; $140 \mathrm{mM}$ $\mathrm{NaCl}, 7.2 \mathrm{mM}$ glucose, $3 \mathrm{mM} \mathrm{KCl}, 1 \mathrm{mM} \mathrm{MgCl} 2,1.2 \mathrm{mM}$ $\mathrm{CaCl}_{2}, 1.2 \mathrm{mM} \mathrm{Na} \mathrm{HPO}_{4}, 0.27 \mathrm{mM} \mathrm{Na}_{2} \mathrm{HPO}_{4}, \mathrm{pH} 7.4$ ) at $2 \mu \mathrm{l} / \mathrm{min}$. An equilibration period of $30 \mathrm{~min}$ was allowed to recover basal levels of extracellular DA in striatum, and then dialysates were collected every $20 \mathrm{~min}$ in ice-cold tubes containing $5 \mu \mathrm{l}$ of $0.2 \mathrm{M}$ perchloric acid. After $60 \mathrm{~min}$, ACSF was replaced with high-potassium ACSF $(93 \mathrm{mM} \mathrm{NaCl}$ and $50 \mathrm{mM} \mathrm{KCl}$ ), to induce neurotransmitter release, and a further 3 samples were collected, after which the physiological ACSF was infused again. Each sample was snap frozen in dry ice and stored at $-80{ }^{\circ} \mathrm{C}$ until HPLC analysis was performed as described above. Mice were sacrificed and the microdialysis probe position was verified in the striatum. For each individual mouse, DA release was normalized to the baseline fraction ( $0 \mathrm{~min}$ ) and expressed as fold difference relative to the average DA release directly following $\mathrm{K}^{+}$stimulation (60 min fraction) in the respective control C57Bl/6S group. DA release was compared between $\mathrm{MI} 2$ and $\mathrm{C} 57 \mathrm{Bl} / 6 \mathrm{~S}$ mice and between anle138b- and placebo-treated mice.

\section{Stereological counting of nigral cells}

Six $30 \mu \mathrm{m}$-thick coronal sections, evenly spaced at $180 \mu \mathrm{m}$ within the range covering the whole $\mathrm{SNpc}$ (between $-2.8 \mathrm{~mm}$ and $-3.85 \mathrm{~mm}$ AP from bregma [28]), were investigated in each mouse. TH immunohistochemistry was performed as described above, and tissue sections were analyzed with the optical fractionator probe using Stereo Investigator 11.07 (MBF Bioscience) with Olympus BX53 microscope equipped with QImaging Retiga camera and X-Y step motorized stage controlled by MAC 6000 System 


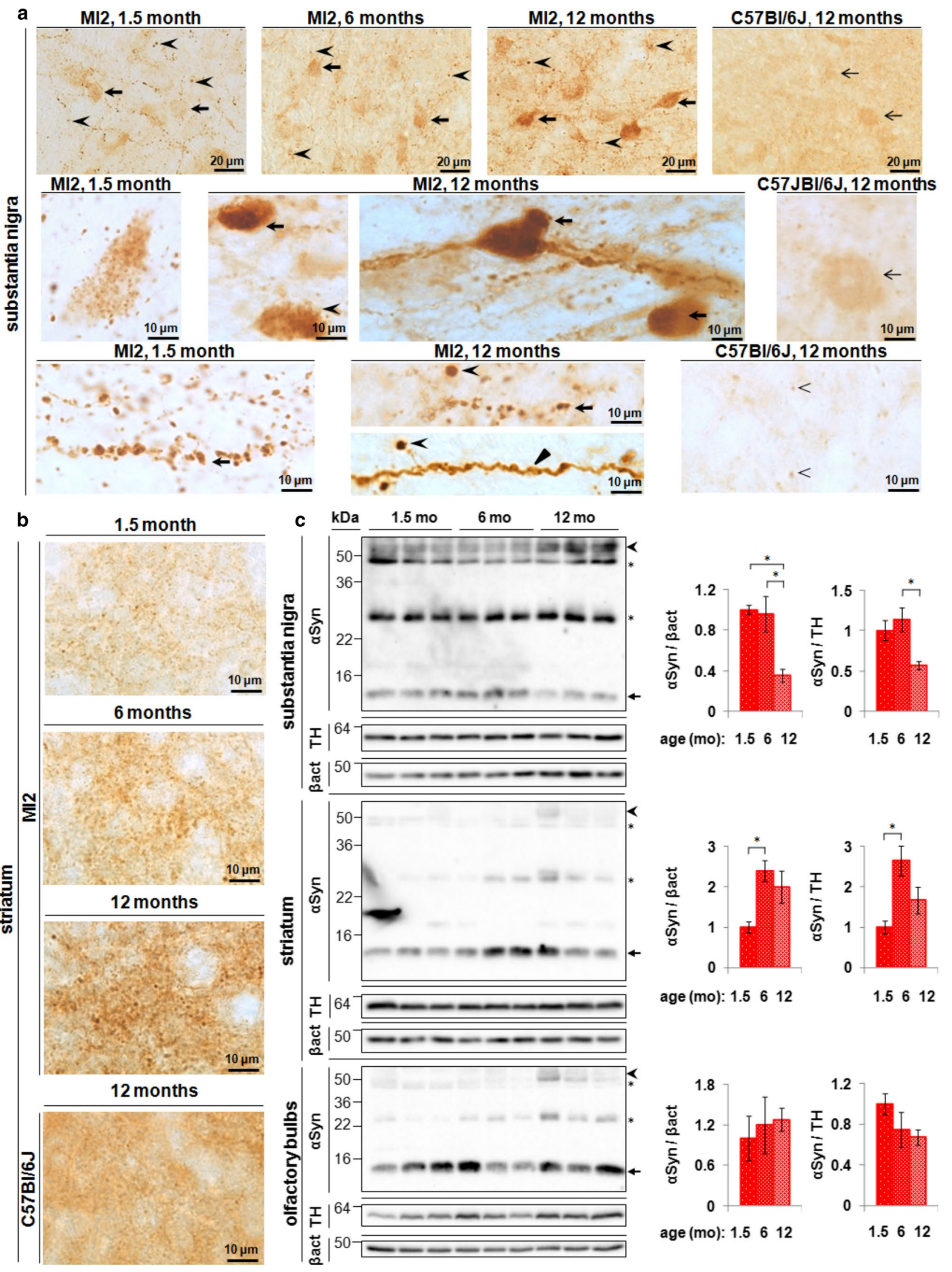


4Fig. 2 Aggregation of $1-120 \mathrm{~h} \alpha$ Syn protein in MI2 mice. a Top panel: progressive accumulation of $1-120 \mathrm{~h} \alpha \mathrm{Syn}$ protein with age in SNpc of MI2 mice in cell bodies (arrows) and processes (arrowheads). Middle panel: abundant small inclusions of 1-120 hoSyn protein in SNpc cell bodies are present at 1.5 months and large, LB-like aggregates in SNpc neurons (arrows) among cells with 1-120 hoSyn punctate staining (arrowhead) are found at 12 months of age. Bottom panel: large 1-120 hoSyn puncta are distributed along the processes in SNpc at 1.5 and 12 months of age (arrows). At 12 months of age, processes uniformly filled with 1-120 hoSyn (triangle) and round inclusions containing condensed 1-120 hoSyn protein (arrowheads) are also visible. The staining of $\alpha$ Syn in WT C57B1/6J mice is much less intense and more homogenous. Middle right panel: less $\alpha \mathrm{Syn}$ is present in cell bodies, and no cellular inclusions are found (arrow) in control mice. Bottom right panel: $\alpha$ Syn puncta in the nigral processes are much less numerous and smaller in C57B1/6J mice than in MI2 mice (arrowheads), (see also Supplementary Fig. S2, Online Resource 1). b Progressive accumulation of 1-120 hoSyn puncta in MI2 striatal neuropil in 1.5, 6 and 12 month-old MI2 mice. In 12 month-old $\mathrm{C} 57 \mathrm{Bl} / 6 \mathrm{~J}$ mice striatal $\alpha \mathrm{Syn}$ is distributed more homogenously and large $\alpha$ Syn-positive puncta are not present (bottom panel). c Immunoblotting of brain lysates from MI2 mice as a function of age. The levels of monomeric 1-120 hoSyn ( 14 kDa, arrow) shown in the western blots were quantified and normalized to either $\beta$-actin or TH (right panels). Data are expressed as fold difference compared to 1.5 month-old animals (mean \pm SEM, $n=3$ mice, one-way ANOVA, multiple comparison with Bonferroni corrections). In SN, a reduction of monomeric 1-120 hoSyn was found as a main effect of age [statistically significant differences between 1.5 and 12 months and between 6 and 12 months ( $\alpha$ Syn/ $\beta$-actin or $\alpha$ Syn/ $\mathrm{TH} ; * p<0.05)]$ for all comparisons. In the striatum there was a significant increase of 1-120 h $\alpha$ Syn between 1.5 and 6 months of age $(* p<0.05)$ for both $\alpha \mathrm{Syn} / \beta$-actin and $\alpha \mathrm{Syn} / \mathrm{TH}$. There was no significant change in OB. In all the three brain regions increased amounts of high molecular weight (HMW) 1-120 hoSyn bands ( 55 kDa, arrowhead) were present in MI2 mice at 12 months compared to 1.5 and 6 months of age (see also Supplementary Fig. S3, Online Resource 1). Stars denote the non-specific bands recognized by Syn1 antibody in both MI2 and $\alpha$ Syn-null C57B1/6S mice [29] (see detailed statistical evaluation in Online Resource)

controller. The SNpc area was outlined under a 4x objective and cells were counted under a $100 \times$ objective using the following parameters: counting grid size $-220 \times 220 \mu \mathrm{m}$, counting frame $-85 \times 85 \mu \mathrm{m}$. The thickness of the section was measured at each counting site. Data were processed using built-in software and the optical fractionator formula to estimate the total number of TH-positive neurons in SNpc.

In order to confirm neurodegeneration in SNpc of MI2 mice, stereological counting of all nigral neurons was performed after staining the sections with the NeuN panneuronal marker. In order to contour $\mathrm{SNpc}$ for NeuN cell counting, TH immunofluorescence was performed in the same sections and staining visualized with Alexa Fluor 488 secondary antibody. Sections were visualised at $488 \mathrm{~nm}$ using an epifluorescent microscope and the SNpc was outlined based on TH immunofluorescence. The resulting contour was super-imposed in the bright field image of NeuN immunohistochemistry. The slides were transferred to a stereology microscope, and the contour of $\mathrm{SNpc}$ was manually reconstructed in bright field in Stereo Investigator 11.07 before stereological counting of NeuN-positive cells (see also Supplementary Fig. S6, Online Resource 1).

\section{Measurements of $\mathrm{TH}^{+}$striatal innervation}

Density of dopaminergic fibers in the striatum was estimated by the modified spherical probe counting method [26] in two $30 \mu \mathrm{m}$-thick coronal sections, representing medial and caudal striatum $(+0.12 \mathrm{~mm}$ and $-0.66 \mathrm{~mm}$ AP from bregma [28]). The tissue was processed for TH immunohistochemistry as described above and analyzed using Stereo Investigator 11.07. A series of hemispherical ( $10 \mu$ m radius) probes, arranged in a 2 dimensional array, spaced at $250 \mu \mathrm{m}$ intervals was superimposed on the striatum, and the total number of TH-positive fibers that cross the surface of the probe was counted under a $100 \times$ objective. The thickness of the section was measured at each counting site. The estimated total length of TH-positive fibres in individual sections was normalized to the measured volume of the section and averaged between counting sites. Data are expressed as nm of length of TH-positive fibers in $\mu^{3}$ of striatal volume.

\section{Rotarod test}

Motor performance was tested using an accelerating rotarod (Ugo Basile). The test was performed in mice at 6,12, 15 and 20 months of age using separate cohorts of animals at each time point. Both males and females were tested in MI2 and C57B1/6S groups, but we observed that some 15 and 20 month-old MI2 females were overweight, therefore, since the body weight may affect motor performance [20, 24], the heaviest individuals from this group were excluded from the test, to obtain no significant difference between the MI2 and C57B1/6S females mice tested ( $t$ test, $p=0.552$ at 15 months, $p=0.851$ at 20 months). Prior to the test, mice were trained for 2 days. Each training session consisted of four trials at constant speed of $16 \mathrm{rpm}$, for a maximum of $60 \mathrm{~s}$ per trial. Mice were then tested using the acceleration mode (5-40 rpm) for three consecutive days with three trials per day and with at least 30 min intervals between the trials. Mice were placed on the rod and the time that the individual mouse took to fall from the rod was measured. Single trials were considered as technical replicates, and an average of 9 technical replicates ( 3 days per 3 trials) was calculated for each animal to obtain each biological replicate that was then used for statistical purposes.

\section{Static rod test}

The cohort of 20 month-old mice used for the rotarod test were then used for the static rod test performed according to the method of Deacon with modifications [9]. Two $60 \mathrm{~cm}$ long rods of $25 \mathrm{~mm}$ and $15 \mathrm{~mm}$ diameters were attached to 
the platform and held horizontally $30 \mathrm{~cm}$ above the cushioned surface. Mice were placed at the end of the rod, facing away the platform and were allowed to freely turn back and walk to the platform. Prior to the test animals received training trials using $25 \mathrm{~mm}$ and $15 \mathrm{~mm}$ rods. On the next day, mice were tested on both $25 \mathrm{~mm}$ and $15 \mathrm{~mm}$ rods (one trial per each diameter). Two parameters were measured: orientation time (time taken to turn from the initial position towards the platform) and transit time (time taken to traverse the rod from the far end to the platform).

\section{Gait assessment with the DigiGait system}

In order to detect the presence of early motor behavior during the progression of $\alpha$ Syn aggregation and synaptic dysfunction in MI2 mice, we used DigiGait automated gait imaging system (Mouse Specifics Inc.) [14, 27]. MI2 and control C57B1/6S mice were tested at 3, 6, 9, 12, 15 and 18 months of age. Male mice were selected because at older age some female MI2 mice appeared overweight and this could have influenced the results. Individual animals were weighed before the analysis, and placed on the transparent motorised treadmill, that was set at $10 \mathrm{~cm} / \mathrm{s}$, the speed was adapted to the performance of aged animals (up to 18 months) that were still able to walk steadily at this rate but not at higher speed. The camera placed under the belt captured the gait of each mouse individually at 150 frames/s, and 3-5 s-long video of steady walking was then selected for further analysis for each mouse. The videos were analyzed by the DigiGait software, that measured the area of paw-belt contact in subsequent frames of the video, and plotted it against time, producing a plot representing stepping cycles for each individual paw. The data were then automatically processed by the DigiGait software to calculate indices for individual paws. These results were analyzed after averaging fore right with fore left paw values and hind right with hind left paw for individual mice.

After the detection of a motor phenotype at early ages the test was repeated to determine whether anle $138 \mathrm{~b}$ had an effect on the motor behavior detected. However, because the number of male mice available at the correct age was not sufficient for statistically significant conclusions we combined males and females taking care to select mice of similar weight. MI2 and C57Bl/6S animals treated with either anle $138 \mathrm{~b}$ or placebo diet were tested at 9 months of age ( 1 day prior to the treatment start), at 10.5 months of age (in the middle of the treatment period), and at 12 months of age ( 1 day prior to the treatment end).

\section{Anle138b treatment}

Mice were treated from 9 to 12 months of age with anle138b, starting at a time when loss of striatal DA release was already present in MI2 mice but prior to significant nigral neuron loss and completed when MI2 mice presented with no more than $30 \%$ cell loss. Drug or placebo was administered in standard diet $(2 \mathrm{~g} / \mathrm{kg}$ of food, ssniff Spezialdiäten $\mathrm{GMbH})$ throughout the whole 3 months study period to different cohorts of MI2 and C57B1/6S mice. The levels of anle138b in plasma and brains of mice following this type of treatment have been previously reported [36]. At 12 months of age, mice were assessed using in vivo microdialysis. Subsequently, brains were collected for analysis, as described above.

\section{Statistical analysis}

The results are expressed as mean \pm SEM. A $t$ test was used for comparisons between two experimental groups. When more than two means were compared, one-way or two-way ANOVA, followed by a Bonferroni correction for multiple comparisons was used. Data from the in vivo microdialysis experiments were analyzed using two-way or three-way mixed ANOVA, with sampling time point as within-subject factor.

\section{Results}

\section{Generation of 1-120 haSyn MI2 mouse line}

MI2 mice express truncated 1-120 h $\alpha$ Syn under the control of the rat TH promoter (Fig. 1a), but no endogenous mouse $\alpha$ Syn due to a spontaneous deletion of the SNCA gene in the C57Bl/6OlaHsd (C57Bl/6S) mice [31, 35]. A C-terminally truncated form of $\alpha$ Syn was used because it has been shown to aggregate faster than the full-length protein [7] and its presence has been shown in brain extracts from Parkinson's and dementia with Lewy bodies patients [1]. Immunoblotting revealed the presence of the transgenic protein in brain regions and neurons with TH-expression, such as substantia nigra (SN), striatum and olfactory bulb (OB), while no transgenic protein was expressed in cortex or cerebellum, where TH expression is not prominent (Fig. 1b). Expression of transgenic 1-120 hoSyn appeared significantly lower than that of endogenous $\alpha$ Syn in wild-type (wt) C57B1/6J mice (Fig. 1c) possibly because $\alpha \mathrm{Syn}$ is present in various neuronal populations in wt mice, while its expression in MI2 mice is limited to $\mathrm{TH}^{+}$neurons.

By immunohistochemistry, the distribution of 1-120 hoSyn also followed the TH expression pattern, staining being evident in $\mathrm{SNpc}$ and ventral tegmental area (VTA) neurons, while no staining was evident in the SN pars reticulata, where $\alpha \mathrm{Syn}$ is instead found in wt C57B1/6J mice (Fig. 1d). In the SNpc 1-120 hoSyn expression was localized in both neuronal cell bodies and processes, while 
in the striatum, where nigral neurons project, it appeared as diffuse punctate staining (Fig. 1d).

\section{Progressive aggregation of transgenic 1-120 haSyn}

Immunohistochemical analysis of 1-120 hoSyn in the nigrostriatal system of MI2 mice showed an age-dependent progressive accumulation of the protein. At 1.5 months, anti$\alpha$ Syn antibodies stained small puncta that were diffusely distributed throughout the cell body of nigral neurons as well as numerous swollen processes. A more intense pattern was observed at 6 months, and at 12 months of age when, besides the punctate $\alpha$ Syn immunoreactivity, large LB-like structures with condensed $\alpha$ Syn staining were seen, and neuronal processes were uniformly stained, with some containing large 1-120 hoSyn inclusions (Fig. 2a). None of these features were present in wt $\mathrm{C} 57 \mathrm{Bl} / 6 \mathrm{~J}$ mice, where at 12 months of age, normal $\alpha$ Syn puncta were much smaller and less frequent than the inclusions in the MI2 mice (Fig. 2a).

In the striatum the distribution of 1-120 hoSyn was consistent with its synaptic enrichment, confirmed by co-staining with synaptobrevin (VAMP2), (Supplementary Fig. S1a, Online Resource 1) and followed the pattern of nigrostriatal projections. Staining for transgenic human 1-120 hoSyn appeared as small puncta, accompanied by larger inclusions, whose number increased with age (Fig. 2b), while wild type C57Bl/6J mice displayed a normal diffuse and homogeneous $\alpha$ Syn punctate pattern (Fig. 2b). No $\alpha$ Syn staining was present in wt $\mathrm{C} 57 \mathrm{Bl} / 6 \mathrm{~S}$ mice with deletion of the endogenous $\alpha$ Syn gene. Staining for VAMP2 showed a progressive redistribution of the protein at the synapse (Supplementary Fig. S1b, Online Resource 1), a pattern similar to that previously reported in our $\alpha$-Syn120 mice showing SNARE protein redistribution [12].

Staining with anti-ubiquitin antibody was present in some large $\alpha$ Syn aggregates in the neurons of the SNpc starting from 12 months of age in MI2 mice (Supplementary Fig. S2b, Online Resource 1), but was not detected at any age in $\alpha$ Syn presynaptic aggregates in the striatum (data not shown). TfT and pFTAA did not show any specific staining (data not shown). PK digestion of tissue sections from $\mathrm{SNpc}$ and striatum of MI2 mice at 1.5 and 12 months of age showed that, while in the SNpc some of $\alpha$ Syn aggregates were resistant to PK treatment and still detectable following treatment, no $\alpha$ Syn staining was found in the striatum indicating that aggregates had been fully digested in this region or if present they were too small to be detected with conventional microscopy (Supplementary Fig. S2a, Online Resource 1).

Immunoblotting of SN extracts showed a significant reduction of monomeric 1-120 hoSyn in 12 month-old MI2 compared with 6 and 1.5 month-old MI2 mice with an associated increase in higher molecular weight (HMW) 1-120 hoSyn species (Fig. 2c). In the striatum, monomeric
1-120 hoSyn increased between 1.5 and 6 months of age and an increase in HMW species was present at 12 months of age, similar to the SN (Fig. 2c).

Serial extraction of $\alpha$ Syn with buffers of increasing strength from both $\mathrm{SNpc}$ and striatum showed an increase in the presence of $\alpha \mathrm{Syn}$ in TritonX-insoluble fractions between 1.5 and 12 months of age, suggesting increasing aggregates insolubility (Supplementary Fig. S3, Online Resource 1).

No apparent difference in monomeric 1-120 hoSyn was found in the OB between 1.5-, 6- and 12 months of age, although an increase in HMW bands was also present in this region (Fig. 2c).

\section{Super-resolution imaging of striatal 1-120 haSyn}

To obtain a more detailed analysis of synaptic 1-120 hoSyn at a near single-molecule resolution, we imaged the transgenic protein in the striatum of mice at 1.5, 6 and 12 months of age using $d$ STORM following immunofluorescence staining with a human $\alpha$ Syn specific antibody (Fig. 3a). To perform quantitative analysis of the aggregation profile, size, shape, inner density, local density per region of interest and percentage of aggregated 1-120 hoSyn, a semi-automated software was built that implements a novel set of meta-analysis clustering algorithmic tools for analyzing super-resolution data (Supplementary Fig. S4, Online Resource 1). Using this approach, we observed progressive aggregation of 1-120 $\mathrm{h} \alpha \mathrm{Syn}$ and followed the size distribution of the aggregates over time in the striatum of 1.5, 6 and 12 month-old MI2 mice. No specific staining was observed by $d$ STORM in C57B1/6S control mice (see also Supplementary Fig. S3, Online Resource 1) The population of aggregates was divided into 4 groups according to size: small, with a diameter ranging from 20 to $100 \mathrm{~nm}$, medium, with a diameter between 100 and $300 \mathrm{~nm}$, large, with a diameter of 300-500 nm and very large, with a diameter above $500 \mathrm{~nm}$. $d S T O R M$ imaging and analysis demonstrated an age-dependent increase in the overall number of 1-120 hoSyn aggregates from 1.5 to 6 and 12 months of age (Fig. 3b). This increase was mainly due to an age-dependent increase in small (20-100 nm), medium $(100-300 \mathrm{~nm})$ and large $(300-500 \mathrm{~nm})$ aggregates. The number of very large aggregates $(>500 \mathrm{~nm})$ did not significantly change with age (Fig. 3c).

\section{Dopaminergic dysfunction in the striatum of MI2 mice}

To establish whether the progressive aggregation of 1-120 h $\alpha$ Syn affected DA neurotransmission in the striatum in MI2 mice, the total content of striatal DA was measured at 3,6 and 12 months of age. No difference was detected between MI2 and C57B1/6S control mice at 3 and 6 months of age, while a significant reduction in total DA levels was present at 12 months (control, 65.3 \pm 5.8 , MI2, 

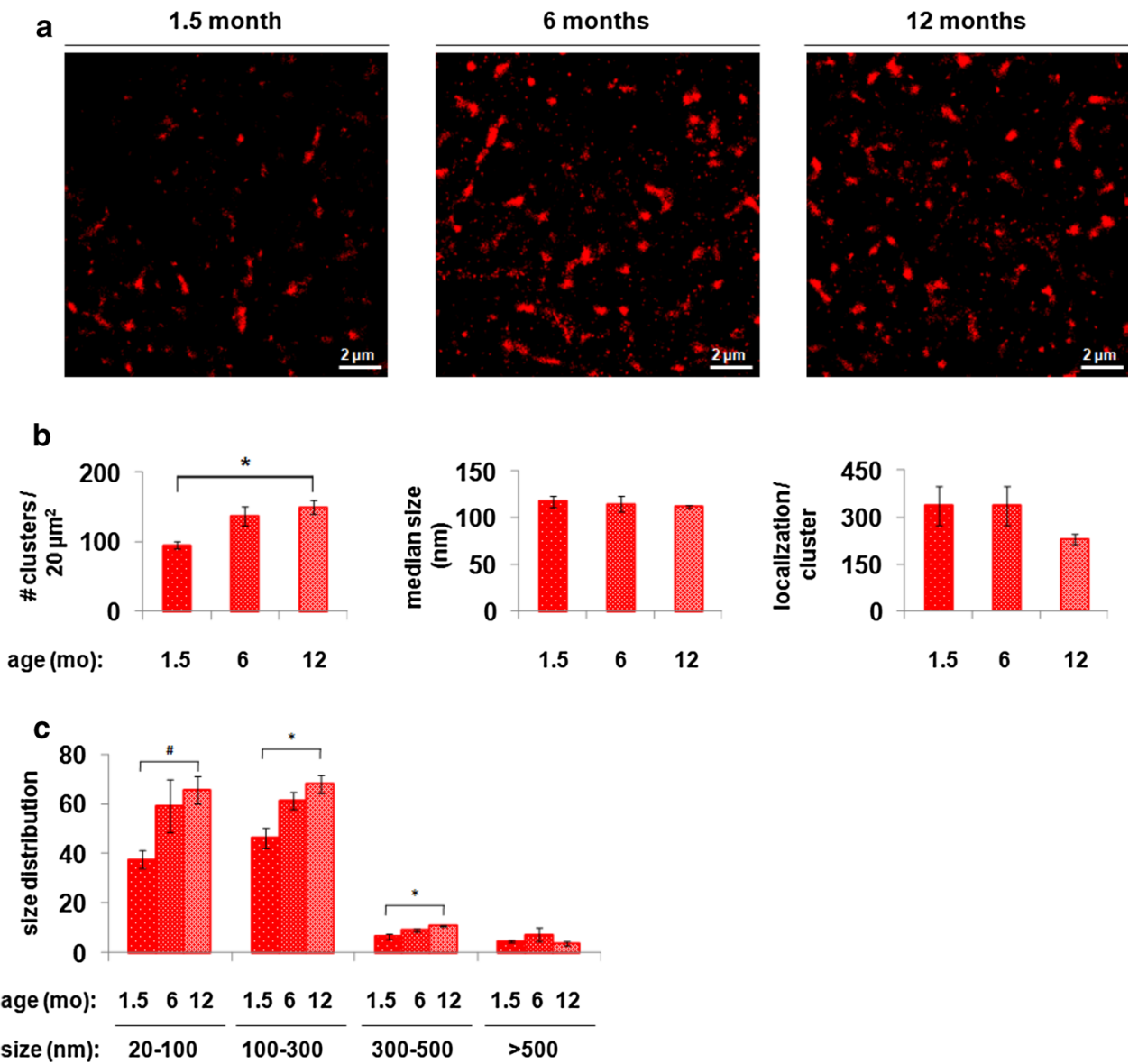

Fig. $3 d$ STORM analysis of the progression of 1-120 h $\alpha$ Syn aggregation in the striatum of MI2 mice. a Representative $d$ STORM images of $1-120 \mathrm{~h} \alpha$ Syn staining in the striatum of MI2 mice at 1.5 , 6 and 12 months of age showing increasing number of aggregates with age. b Quantification of $d$ STORM data (see also Supplementary Fig. S4) (mean \pm SEM, $n=3$ mice, one-way ANOVA, multiple comparisons with Bonferroni correction). The main effect of age is the increasing number of aggregates (clusters) $(* p<0.05)$. No differences in the aggregate median size or number of localizations per

$38.1 \pm 5.7 \mathrm{pmol} \mathrm{DA} / \mathrm{mg}$ of tissue, Fig. 4a). DA content in 12 month-old MI2 mice was also reduced compared to MI2 mice at 3 and 6 months of age $(87 \pm 9.2$ and $73.1 \pm 11.1 \mathrm{pmol}$ $\mathrm{DA} / \mathrm{mg}$ of tissue, respectively; Fig. 4a). No difference was found in DA levels at 12 months of age between control mouse lines with $(\mathrm{C} 57 \mathrm{Bl} / 6 \mathrm{~J})$ and without $(\mathrm{C} 57 \mathrm{Bl} / 6 \mathrm{~S})$ endogenous $\alpha$ Syn (Supplementary Fig. S5a, Online Resource 1), confirming that the absence of endogenous $\alpha$ Syn does not affect striatal DA levels, as previously shown [13].

Dopaminergic deficit in MI2 mice was further investigated by measuring striatal DA release using in vivo microdialysis. DA release was induced in freely moving mice by infusion of $50 \mathrm{mM} \mathrm{K}^{+}$. No difference in DA release was observed between MI2 and control C57Bl/6S mice at 3 months of age, while at 6 months, a significant reduction in $\mathrm{K}^{+}$stimulated DA release was present in MI2 mice cluster (e.g. the inner density which measures the number of fluorescent flashes in a cluster) were found. c Analysis of cluster size distribution shows a statistically significant increase in the number of small-size aggregates (20-100 nm, $t$ test $\left.{ }^{\#} p<0.05\right)$, medium size aggregates $(100-300 \mathrm{~nm}$, one way ANOVA, $* p<0.05)$ and large-size aggregates (300-500 nm, one way ANOVA, $* p<0.05$ ), between 1.5 and 12 months of age (see detailed statistical evaluation in Online Resource)

compared with $\mathrm{C} 57 \mathrm{~B} 1 / 6 \mathrm{~S}$ controls [samples were measured at 60,80 and $100 \mathrm{~min}$, normalized to peak $\mathrm{C} 57 \mathrm{Bl} / 6 \mathrm{~S}$ value (at $60 \mathrm{~min})](0.57 \pm 0.15$ vs $1 \pm 0.08$ at $60 \mathrm{~min}, 0.38 \pm 0.09$ vs $0.63 \pm 0.07$ at $80 \mathrm{~min}$, and $0.34 \pm 0.03$ vs $0.54 \pm 0.05$ at $100 \mathrm{~min}$ in MI2 vs control mice; Fig. 4b). This impairment was progressive, with the loss of induced DA release being more prominent at 9 months of age $(0.24 \pm 0.09$ vs $1 \pm 0.25$ at $60 \mathrm{~min}, 0.17 \pm 0.06$ vs $0.71 \pm 0.17$ at $80 \mathrm{~min}$, and $0.17 \pm 0.06$ vs $0.6 \pm 0.14$ at $100 \mathrm{~min}$ in MI2 vs control mice) and further exacerbated at 12 months of age $(0.16 \pm 0.06 \mathrm{vs}$ $1 \pm 0.24$ at $60 \mathrm{~min}, 0.13 \pm 0.05$ vs $0.87 \pm 0.2$ at $80 \mathrm{~min}$ and $0.11 \pm 0.04$ vs $0.61 \pm 0.17$ at $100 \mathrm{~min}$ in MI 2 vs control mice; Fig. $4 \mathrm{~b})$. No difference was observed in striatal DA release between $\mathrm{C} 57 \mathrm{Bl} / 6 \mathrm{~S}$ and $\mathrm{C} 57 \mathrm{Bl} / 6 \mathrm{~J}$ control mice at 12 months of age (Supplementary Fig. S5b, Online Resource 1). These findings indicate that striatal DA release impairment in 

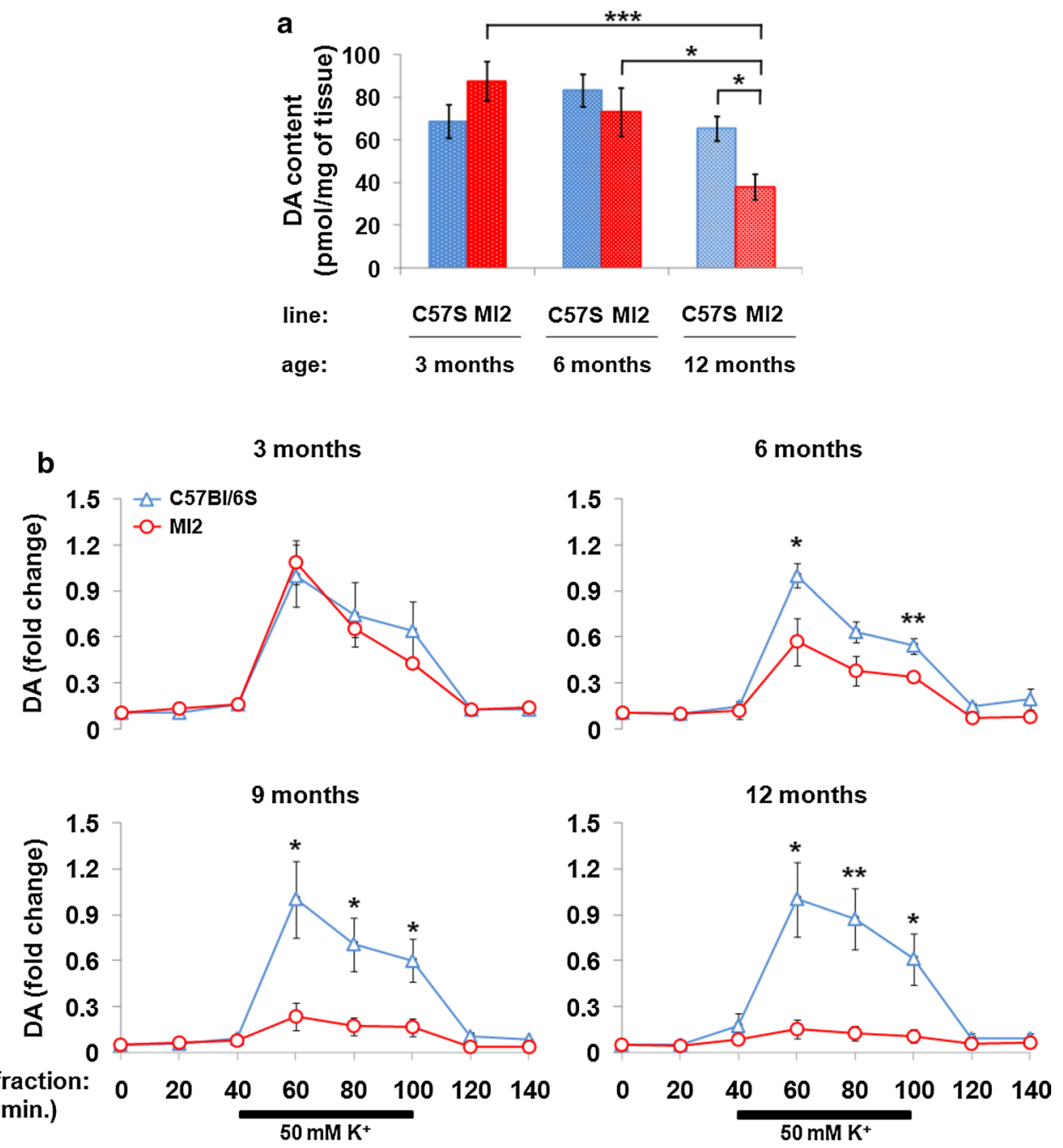

Fig. 4 Striatal dopaminergic deficit in MI2 mice. a DA was measured in striatal lysates of MI2 and C57B1/6S mice at 3, 6 and 12 months of age (mean \pm SEM, $n=5-8$ mice per group). A main effect of age and interaction between genotype and age was identified by two-way ANOVA with Bonferroni correction, showing a significant reduction of DA levels in MI2 mice at 12 months compared to 12 month old C57B1/6S mice, and to 3 month old and 6 month old MI2 mice $(* p<0.05, * * * p<0.001)$ (see also Supplementary Fig. S5a, Online Resource 1 and for DDPAC content S5c). b Striatal DA release was measured by in vivo microdialysis following the infusion of $50 \mathrm{mM}$ $\mathrm{KCl}$ for $60 \mathrm{~min}$ between 40 and $100 \mathrm{~min}$ of the experiment. Data

MI2 mice is associated with the progressive aggregation of 1-120 hoSyn. Importantly, a significant reduction in DA release in MI2 mice appears earlier than a reduction of total DA content in striatal tissue extracts, supporting the presence of a specific synaptic dysfunction (Fig. 4).

\section{Loss of dopaminergic neurons in SNpc of MI2 mice}

Nigral DA neuron death in the MI2 mice was investigated by unbiased stereological counting of cells stained for TH. are expressed as a fold difference compared to the baseline fraction ( 0 min), normalized to the value obtained in age-matched $\mathrm{C} 57 \mathrm{Bl} / 6 \mathrm{~S}$ controls at $60 \mathrm{~min}$ (mean $\pm \mathrm{SEM}, n=4-6$ mice). At 3 months of age no difference between MI2 and $\mathrm{C} 57 \mathrm{Bl} / 6 \mathrm{~S}$ mice was observed, but a significant progressive decrease in DA release was found at 6 , 9 and 12 months of age in MI2 compared with C57B1/6S mice (see also Supplementary Fig. S5b, Online Resource 1). Two-way mixed ANOVA revealed a significant interaction between genotype and sample time at 6, 9 and 12 months of age $(* p<0.05, * * p<0.01, t$ test for individual sampling time points) (see detailed statistical evaluation in Online Resource)

The results showed a progressive $\mathrm{TH}^{+}$cell loss in $\mathrm{SNpc}$ of MI2 mice compared to C57B1/6S mice starting at 9 months of age (MI2, 11,174 \pm 825.1 vs control, 13,222 \pm 1658.8 $\mathrm{TH}^{+}$neurons) becoming significant at 12 months of age [31\% reduction in MI2 vs control mice: $8530.4 \pm 751.9$ (MI2) vs $12,398.8 \pm 946.1(\mathrm{C} 57 \mathrm{Bl} / 6 \mathrm{~S})]$ and further progressed at 20 months of age [ $54 \%$ reduction in MI2 vs controls: $6345.3 \pm 523.1$ (MI2) vs 13,836 $\pm 616.1(\mathrm{C} 57 \mathrm{Bl} / 6 \mathrm{~S})$ $\mathrm{TH}^{+}$neurons, respectively] (Fig. 5a, b). The number of $\mathrm{TH}^{+}$cells in $\mathrm{SNpc}$ of 20 month-old MI2 mice was also 

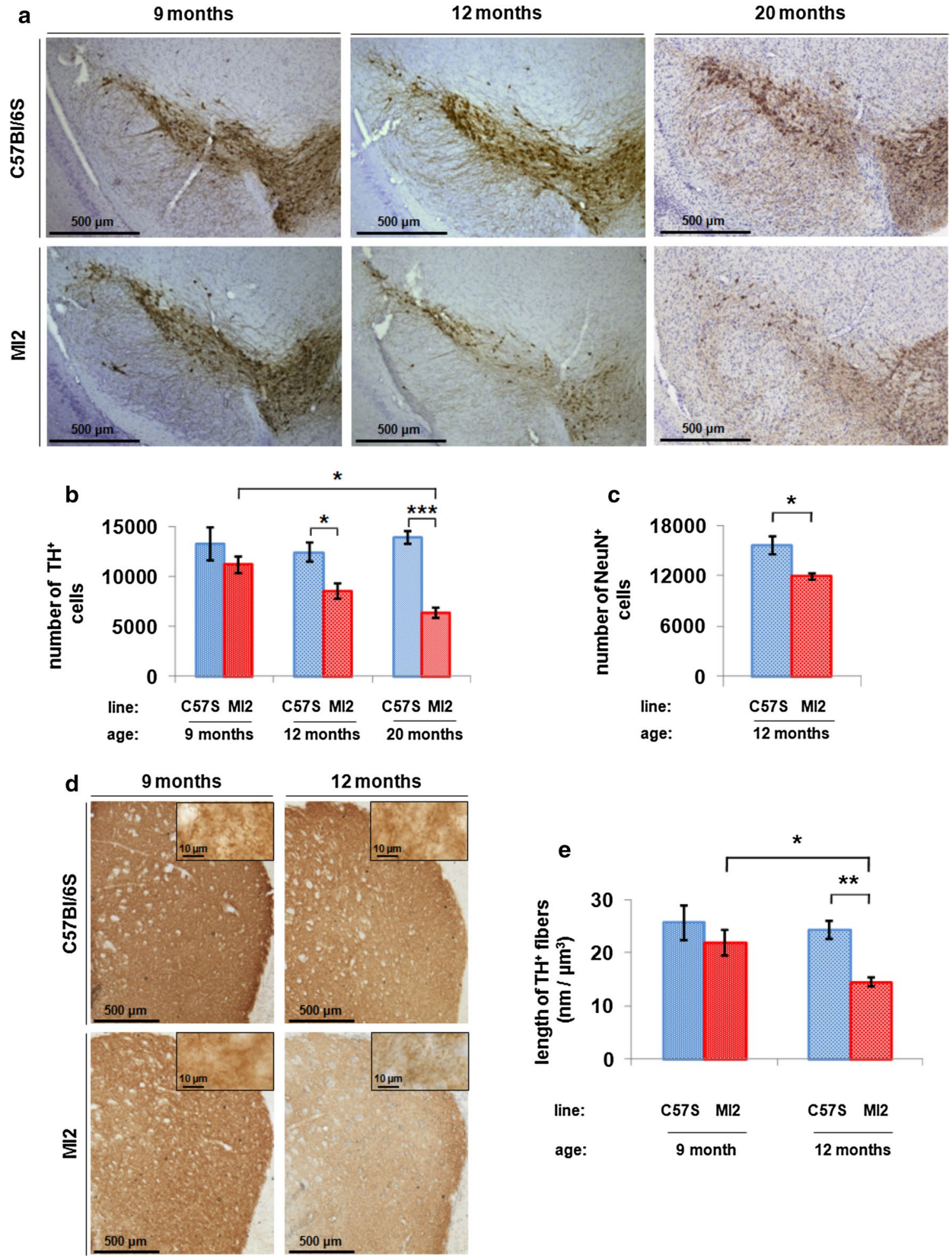

significantly lower (by 43\%) compared to that in 9 monthsold MI2 mice (Fig. 5b).

To confirm neuronal loss in SNpc and rule out the possibility that the decrease in $\mathrm{TH}$ cell number was reflecting a possible reduction in $\mathrm{TH}$ expression, cell counting was performed in 12 month-old mice following staining with
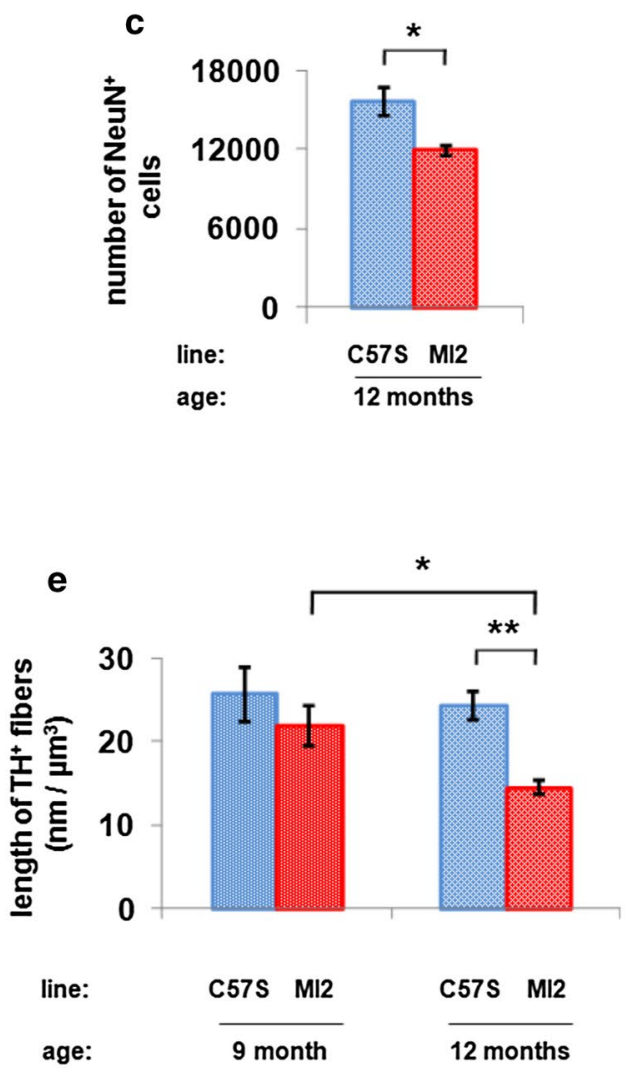

age: the neuronal marker NeuN. NeuN and TH staining images were overlapped to delimit the counting area to the SNpc (Supplementary Fig. S6, Online Resource 1). The results showed a $20 \%$ reduction of $\mathrm{NeuN}$ positive neurons in $\mathrm{SNpc}$ of 12 month-old MI2 mice compared to C57Bl/6S $[11,877 \pm 383.5(\mathrm{MI} 2)$ and 15,633.5 $\pm 1020.7(\mathrm{C} 57 \mathrm{~B} 1 / 6 \mathrm{~S})$ 
४Fig. 5 Loss of dopaminergic neurons in SNpc of MI2 mice. a Immunohistochemistry showed a reduction in TH staining in SNpc of MI2 mice compared to C57B1/6S animals at 12 and 20 months of age. $\mathbf{b}$ Stereological counting of TH-positive neurons in SNpc. Average number of nigral TH-positive cells \pm SEM, $n=4-6$ mice per group; two-way ANOVA with Bonferroni correction showing a significant reduction of $\mathrm{TH}^{+}$neurons in MI2 mice compared to $\mathrm{C} 57 \mathrm{Bl} / 6 \mathrm{~S}$ controls at $12\left({ }^{*} p<0.05\right)$ and $20(* * * p<0.001)$ months of age. There was also a significant reduction in $\mathrm{TH}^{+}$cells in MI2 mice between 9 and 20 months of age. c Stereological counting of total, $\mathrm{NeuN}^{+}$ neuron number in SNpc at 12 months of age (see also Supplementary Fig. S6, Online Resource 1). A significant decrease of $\mathrm{NeuN}^{+}$ neurons is present in MI2 mice compared to $\mathrm{C} 57 \mathrm{~B} 1 / 6 \mathrm{~S}$ mice (average number of nigral NeuN-positive cells \pm SEM, $n=4$ mice per group; $t$ test, $\left.{ }^{*} p<0.05\right)$. d Reduced TH-positive fiber staining in the striatum of MI 2 mice compared to C57Bl/6S mice at 12 months of age. Insets show high magnification images. e $\mathrm{TH}^{+}$striatal neurites were estimated using the spaceball probe (mean length of striatal $\mathrm{TH}^{+}$fibers per volume of striatal tissue \pm SEM, $n=4-6$ mice per group; effect of genotype was identified by two-way ANOVA). Significant decrease in the total length of $\mathrm{TH}^{+}$fibers was present in 12 month-old MI2 mice compared to 12 month-old C57B1/6S mice $(* * p<0.01)$ and 12 and 9 month-old MI2 animals $(* p<0.05)$, multiple comparisons with Bonferroni corrections (see detailed statistical evaluation for $5 \mathrm{~b}, 5 \mathrm{c}$, 5 e in Online Resource)

cells; Fig. 5c]. The reason for the reduced proportional neuronal loss observed following NeuN staining compared to $\mathrm{TH}$ staining is due to the fact that $\mathrm{NeuN}$ recognizes both $\mathrm{TH}$ and non-TH neurons in the SNpc, indeed, the absolute number of cell loss was similar for $\mathrm{TH}$ and NeuN stained neurons ( -3868 and -3757 neurons, respectively).

We then assessed changes in DA innervation in the striatum of MI2 mice at different ages. We observed a significant decrease in striatal $\mathrm{TH}^{+}$innervation at 12 but not at 9 months in MI2 mice compared to C57B1/6S mice (Fig. 5d). In order to determine the loss of striatal innervation, we evaluated the density of TH-positive fibers by estimating their length in a defined volume of striatal tissue. We found that MI2 mice had a $40 \%$ loss of striatal $\mathrm{TH}$ innervation compared to C57Bl/6S mice $\left(14.49 \pm 0.77\right.$ and $24.29 \pm 1.76 \mathrm{~nm}$ per $\mu \mathrm{m}^{3}$, respectively) at 12 months of age while no significant difference was present in 9 month-old MI2 mice compared to controls $\left(21.88 \pm 2.48\right.$ and $25.6 \pm 3.31 \mathrm{~nm}$ per $\mu \mathrm{m}^{3}$, respectively; Fig. 5e). These results indicate that similar to the loss of nigral DA neurons, loss of nigrostriatal DA innervation occurs after the functional synaptic deficit, reflected by a reduction in DA stimulated release that starts at 6 months of age.

\section{Motor impairment in MI2 mice}

An accelerating rotarod test was initially used to analyze motor function in MI2 mice at 6, 12, 15 and 20 months of age. An age-related gradual decline in rotarod performance was present in both $\mathrm{C} 57 \mathrm{~B} 1 / 6 \mathrm{~S}$ and MI2 mice, but the reduction was more pronounced in MI2 mice, resulting in a significant loss of latency to fall from the rotarod at
20 months of age compared to their performance at 6 and 12 months of age $(127.8 \pm 14.9 \mathrm{~s}, 20 \mathrm{~m}, 215.8 \pm 9.9 \mathrm{~s}, 12 \mathrm{~m}$, $220.7 \pm 11.5$ s, 6 m; Fig. 6a). The performance of MI2 mice at 20 months of age was also significantly impaired compared to age-matched C57B1/6S controls $(127.8 \pm 14.9 \mathrm{~s}$ and $181.7 \pm 21.6$ s, respectively; Fig. 6a).

The presence of a motor deficit was also tested in 20 month-old $\mathrm{MI} 2$ compared to $\mathrm{C} 57 \mathrm{Bl} / 6 \mathrm{~S}$ mice using the static rod test where the time required to turn $180^{\circ}$ from the initial position (orientation time) and the time required to travel from the far end of the rod to the platform (transit time) were measured. We found no significant differences between transgenic and control mice in either orientation or transit time when a $25 \mathrm{~mm}$ diameter rod was used, but when the test was performed with a $15 \mathrm{~mm}$ rod, although there was no significant difference in the orientation time, the transit time was longer for MI2 mice compared to controls $(12.8 \pm 1.6 \mathrm{~s}$ and $8.1 \pm 0.8 \mathrm{~s}$, respectively), confirming a selective motor impairment in MI2 mice (Fig. 6b).

To determine whether earlier subtle changes were present in motor behavior, we tested MI2 male mice using the more sensitive DigiGait system. The animals were weighed prior to the experiments, and we identified a trend towards increased body weight in MI2 males that however reached the significance only at 18 months of age (Supplementary Fig. S7a, Online Resource 1). The results of the DigiGait assessment show alterations in the gait pattern of MI2 mice in comparison with C57B1/6S animals starting from 9 months of age, and were manifested by decreased forelimb stride length $(4.256 \pm 0.178 \mathrm{~cm}$ vs. $4.756 \pm 0.14 \mathrm{~cm})$ and forelimb stance $(0.303 \pm 0.014 \mathrm{~s}$ vs. $0.334 \pm 0.009 \mathrm{~s})$, swing $(0.123 \pm 0.005 \mathrm{~s}$ vs. $0.141 \pm 0.007 \mathrm{~s})$ and propulsion $(0.191 \pm 0.009 \mathrm{~s}$ vs. $0.219 \pm 0.008 \mathrm{~s})$ duration, that was still present at the end of the test at 18 months of age (Fig. 6c). Impairment of hindlimb gait pattern was less evident, however, a trend towards a decrease in MI2 mice was present at older ages for hindlimb stride length and hindlimb stance and swing duration (Supplementary Fig. S7b, Online Resource 1). Statistically significant differences were found between hindlimb of MI2 mice and C57B1/6S animals in propulsion duration at 18 months $(0.251 \pm 0.01 \mathrm{~s}$ vs. $0.276 \pm 0.005 \mathrm{~s}$ ) (Fig. 6c), and in brake duration at 6 and 18 months (Supplementary Fig. S7b, Online Resource 1).

\section{The oligomer modulator, anle138b, rescues DA deficit in MI2 mice}

MI2 mice reproduce key features of PD, progressive aggregation of $\alpha$ Syn in the nigrostriatal system, progressive impairment of striatal DA release, reduction in total striatal DA, loss of nigrostriatal DA fibers and nigral DA neurons and motor impairment when the SNpc DA neuron loss reaches $50 \%$. This MI2 mouse line, therefore, appears appropriate for testing potential therapies for PD. Hence, 

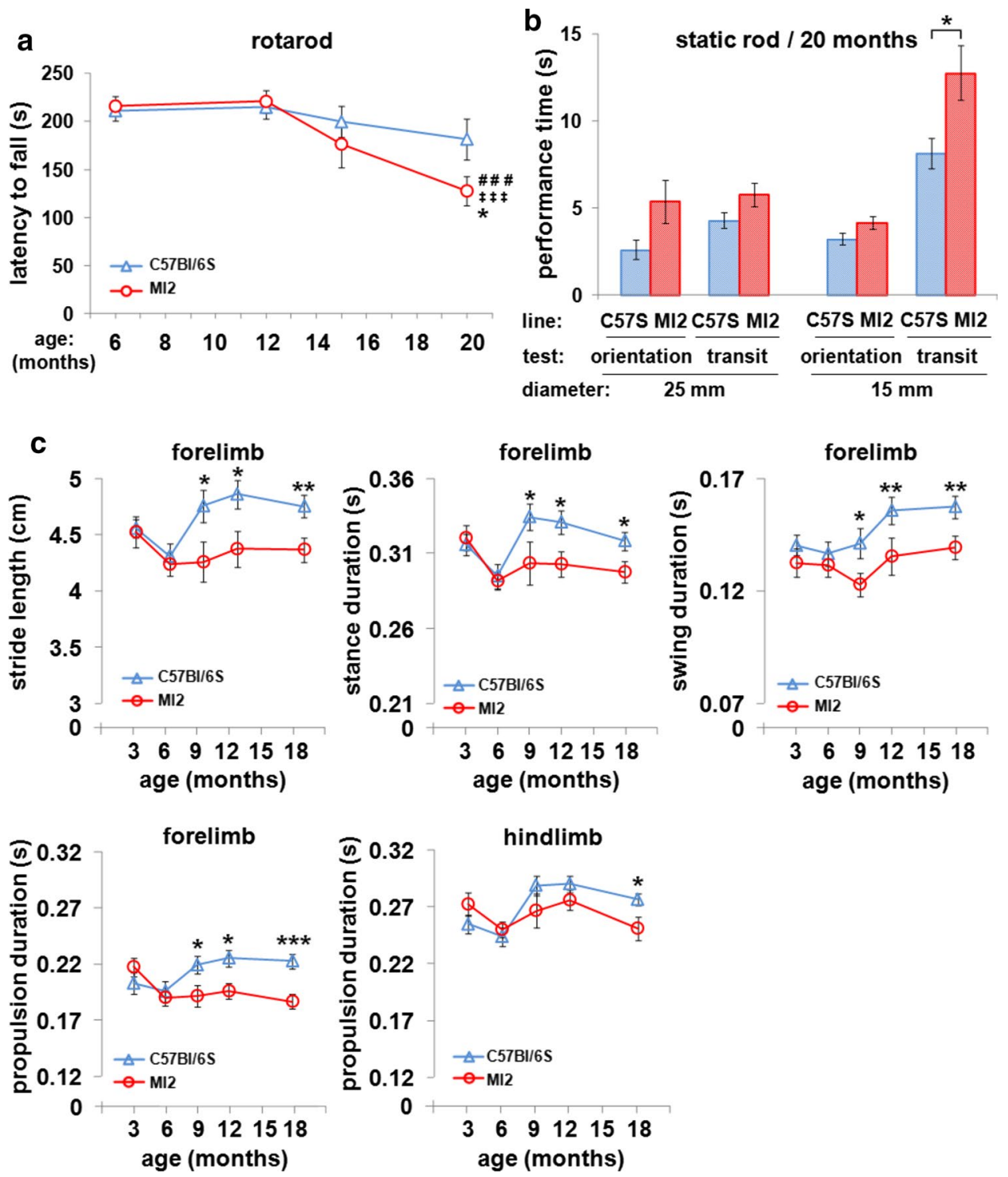

Fig. 6 Motor impairment in MI2 mice. a C57B1/6S and MI2 mice were analyzed using accelerating rotarod test at $6,12,15$ and 20 months of age (mean latency to fall from the rod \pm SEM, $n=12-18$ mice per group). A main effect of age was identified by two-way ANOVA. Statistically significant differences between 6 and 20 months $\left({ }^{\# \#} p<0.001\right)$ and 12 and 20 months $\left({ }^{\ddagger} p<0.001\right)$ in MI2 mice, and between MI2 and C57B1/6S animals at 20 months $\left({ }^{*} p<0.05\right)$ were revealed by multiple comparisons with Bonferroni correction. b Motor performance of 20 month-old mice was tested using the static rod test (mean orientation time or mean transit time \pm SEM, $n=13-18$ mice). No differences were identified between experimental groups using a $25 \mathrm{~mm}$ rod or in orientation time using a $15 \mathrm{~mm}$ rod, but there was a statistically significant difference in transit time on the $15 \mathrm{~mm}$ rod between MI 2 and C57B1/6S mice $\left({ }^{*} p<0.05, t\right.$ test). $\mathbf{c}$ Gait pattern of mice was analyzed between

we investigated if a small molecule modulator of aggregation, anle $138 \mathrm{~b}$ was able to rescue DA impairment in MI2 mice. Furthermore, we used $d$ STORM imaging and
3 and 18 months of age using DigiGait test (see also Supplementary Fig. S7, Online Resource 1). Alterations in individual gait phases were found in MI2 animals compared to control C57B1/6S mice (mean \pm SEM, $n=9-17$ male mice per group). Two-way ANOVA identified a main effect of age on forelimb stance and swing duration, and on hindlimb propulsion duration, a main effect of genotype on forelimb stride length and stance, swing and propulsion duration, and two-way interaction between age and genotype for forelimb propulsion duration. Statistically significant differences were identified at 9 , 12 and 18 months for forelimb stride length and stance, swing and propulsion duration, and at 18 months for hindlimb propulsion duration $(* p<0.05 ; * * p<0.01, * * * p<0.001)$ by pairwise comparisons with Bonferroni correction between MI2 and C57B1/6S mice (see detailed statistical evaluation in Online Resource)

analysis to determine how anle138b acts in vivo and how it affects the spatial distribution of striatal 1-120 hoSyn.

Anle $138 \mathrm{~b}$ was administered in the food $(2 \mathrm{~g} / \mathrm{kg}$ of food) for 3 months to MI2 and C57B1/6S mice. Treatment with 
anle138b or placebo started at 9 months of age, when striatal DA release impairment is already present in MI2 mice, and ended at 12 months of age when $30 \%$ nigral neuron loss is detected in non-treated animals (Fig. 7a). Using immunohistochemistry, we found that anle $138 \mathrm{~b}$ treatment reduced 1-120 hoSyn aggregation in both SNpc cell bodies and synaptic terminals in the striatum (Fig. 7b). Application of $d$ STORM analysis not only confirmed the reduction in striatal $1-120 \mathrm{~h} \alpha$ Syn aggregation but also provided a mechanistic explanation for anle138b activity. $d S T O R M$ analysis revealed two opposite but complementary effects of anle138b: we detected an increased percentage of small, non-clustered 1-120 hoSyn species (anle138b, 53.4 $\pm 2.9 \%$; placebo, $29.9 \pm 3 \%$; Fig. 7 c, d) that occurred concomitantly with a reduction in the inner density of small and large size aggregates (anle138b, 140.9 \pm 12.7 ; placebo, $233.5 \pm 25.3$ fluorophore localizations; Fig. 7c, d). The total number of aggregates was only slightly but not significantly reduced following anle138b treatment (Fig. 7d). Analyses of striatal extracts by immunoblotting confirmed this result showing a reduction in HMW 1-120 hoSyn band and an increase in monomeric protein (Fig. 7e). These data show that anle138b substantially affects the aggregation profile of 1-120 hoSyn in MI2 mice.

Accordingly, we speculated that the non-aggregated small 1-120 hoSyn species, revealed by $d$ STORM, whose number increased following anle138b treatment may represent monomers or small $1-120 \mathrm{~h} \alpha$ Syn assemblies. To examine this hypothesis, we compared the size distribution of the non-aggregated population in MI2 mouse striatum to the size distribution of recombinant monomeric h $\alpha$ Syn. Recombinant h $\alpha$ Syn was plated at low density onto a coverslip, immunolabeled, and imaged using the same protocol as that used to visualize 1-120 hoSyn in MI2 striatal slices. Mapping the recombinant $h \alpha$ Syn population resulted in a size distribution with a mean diameter of $23 \mathrm{~nm}$ and an average of 7 localizations per protein (Supplementary Fig. S8a, b, Online Resource 1). Using the same parameters of clustering, we observed that following anle $138 \mathrm{~b}$ treatment, $85 \%$ of 1-120 hoSyn population in MI2 mouse striatum had a very similar size distribution with a mean diameter of $23 \mathrm{~nm}$ and an average of 7 localizations per protein (Supplementary Fig. S8c, d, Online Resource 1). These data suggest that anle $138 \mathrm{~b}$ treatment resulted in an increase most probably of monomeric and small assemblies of monomeric hoSyn species that could have been possibly either released from dissolving aggregates or not recruited to them.

To determine the effect of anle $138 \mathrm{~b}$ on dopaminergic impairment, we measured striatal DA release in MI2 mice. A 3-month treatment was started at the age of 9 months when the DA release deficit was already present. Released DA was significantly increased in anle138b-treated MI2 mice compared to placebo-treated MI2 animals [Fig. 8a; $\mathrm{K}^{+}$-stimulated DA release, relative values of DA release in anle138b- vs placebotreated MI2 mice sampled at 60, 80 and 100 min, after normalized to peak $\mathrm{C} 57 \mathrm{~B} 1 / 6 \mathrm{~S}$ mouse fraction value (at $60 \mathrm{~min}$ ) reached $0.69 \pm 0.14$ vs $0.13 \pm 0.02(60 \mathrm{~min}), 0.36 \pm 0.07$ vs $0.07 \pm 0.01(80 \mathrm{~min})$ and $0.28 \pm 0.05$ vs $0.09 \pm 0.02$ (100 min)]. The DA release values in C57Bl/6S anle138bvs placebo treated mice were not altered $[1.03 \pm 0.25$ vs $1 \pm 0.12$ (60 $\mathrm{min}), 0.41 \pm 0.09$ vs $0.55 \pm 0.05(80 \mathrm{~min})$ and $0.38 \pm 0.09$ vs $0.39 \pm 0.03$ (100 min) (Fig. 8a)]. We then analyzed the effect of anle138b treatment on the number of nigral DA neurons and found a significant increase in number of $\mathrm{TH}^{+}$neurons in SNpc of anle138b-treated MI2 animals compared to placebo-treated mice (Fig. 8b), with anle138b preventing cell loss. The number of nigral $\mathrm{TH}^{+}$cells did not differ between $\mathrm{C} 57 \mathrm{~B} 1 / 6 \mathrm{~S}$ mice treated with either placebo or anle138b $\left(10,886.7 \pm 706.5\right.$ and $10,792 \pm 858.9 \mathrm{TH}^{+}$cells, respectively) and anle138b-treated MI2 mice $(9944 \pm 567.5$ $\mathrm{TH}^{+}$cells), while a substantial DA neuron loss was present in placebo-treated MI2 mice $\left(7465 \pm 41.4 \mathrm{TH}^{+}\right.$cells) (Fig. 8c).

To determine whether anle138b treatment can affect motor dysfunction, we tested the mice before, during and at the end of treatment using DigiGait system. Mice were weighed before each test, and we found no effect of treatment on the body weight, what shows that body weight differences didn't contribute to behavioral differences between anle138b- and placebo-treated animals over the course of the experiment. An effect of genotype on the body weight was identified only at 12 months of age when MI2 mice were heavier than controls (Supplementary Fig. S9a, Online Resource 1). We found that anle138b rescued several of the parameters associated with the motor impairment (Fig. 9; Supplementary Fig. S9b, Online Resource 1). We observed that MI2 mice treated with anle138b, in comparison with MI2 mice treated with placebo, exhibited increased forelimb stride length $(4.417 \pm 0.094 \mathrm{~cm}$ vs. $4.025 \pm 0.131 \mathrm{~cm}$, respectively) and stance duration $(0.302 \pm 0.006 \mathrm{~s}$ vs. $0.279 \pm 0.008 \mathrm{~s}$, respectively) at 12 months of age, hindlimb stance duration at 10.5 months of age $(0.31 \pm 0.009 \mathrm{~s}$ vs. $0.275 \pm 0.015 \mathrm{~s}$, respectively), as well as forelimb $(0.201 \pm 0.01 \mathrm{~s}$ vs. $0.168 \pm 0.0125 \mathrm{~s}$, respectively) and hindlimb $(0.262 \pm 0.009 \mathrm{~s}$ vs. $0.226 \pm 0.016 \mathrm{~s}$, respectively) propulsion duration at 10.5 months of age (Fig. 9). When we compared placebo-treated MI2 mice with placebo-treated $\mathrm{C} 57 \mathrm{Bl} / 6 \mathrm{~S}$ mice, the length of forelimb stride at $10.5(4.092 \pm 0.22 \mathrm{~cm}$ vs. $4.659 \pm 0.156 \mathrm{~cm}$, respectively) and $12(4.025 \pm 0.131 \mathrm{~cm}$ vs. $4.576 \pm 0.136 \mathrm{~cm}$, respectively) months of age and hindlimb stride at 10.5 $(3.808 \pm 0.211 \mathrm{~cm}$ vs. $4.345 \pm 0.108 \mathrm{~cm}$, respectively $)$, and $12(3.929 \pm 0.133 \mathrm{~cm}$ vs. $4.429 \pm 0.151 \mathrm{~cm}$, respectively) months of age as well as duration of forelimb stance at $10.5(0.281 \pm 0.015 \mathrm{~s}$ vs. $0.321 \pm 0.01 \mathrm{~s}$, respectively $)$ and $12(0.28 \pm 0.009 \mathrm{~s}$ vs. $0.309 \pm 0.009 \mathrm{~s}$, respectively $)$ months of age, hindlimb stance at $10.5(0.275 \pm 0.015 \mathrm{~s}$ vs. 
a
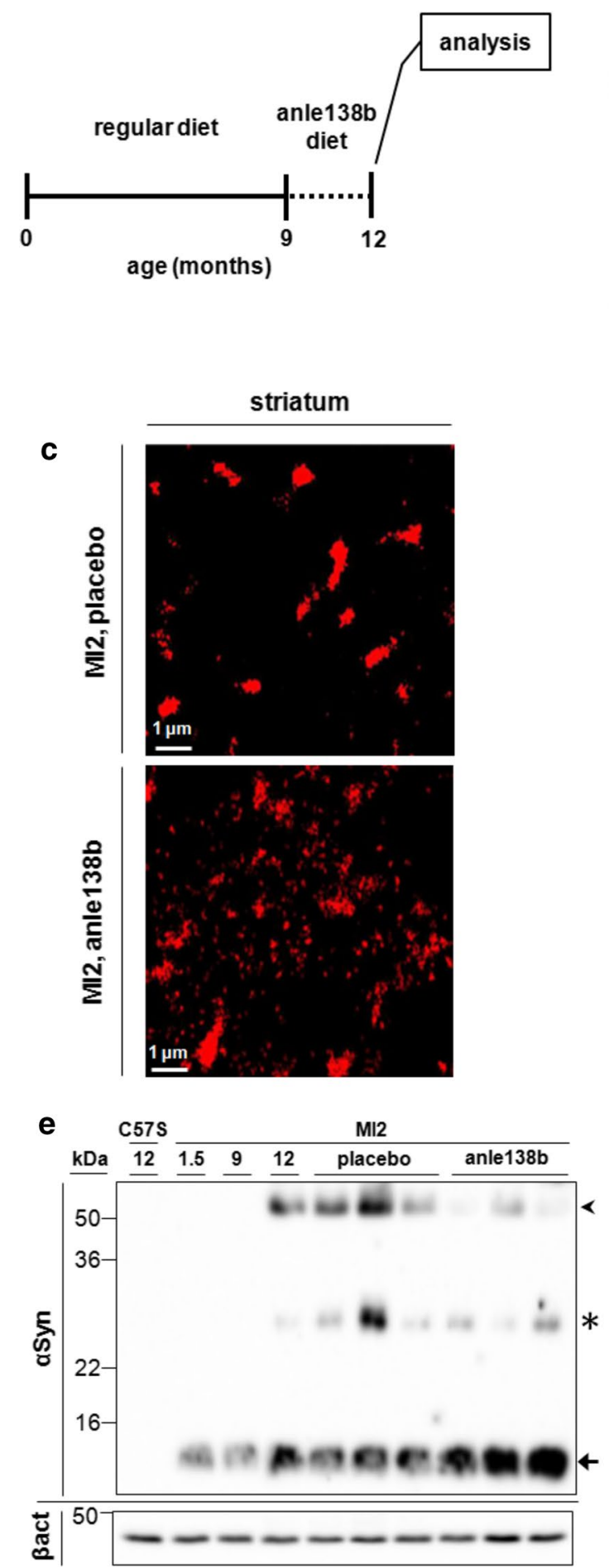

$0.326 \pm 0.008 \mathrm{~s}$, respectively) and $12(0.287 \pm 0.009 \mathrm{~s}$ vs. $0.331 \pm 0.011 \mathrm{~s}$, respectively) months of age, forelimb propulsion at 10.5 months $(0.168 \pm 0.012 \mathrm{~s}$ vs. $0.204 \pm 0.009 \mathrm{~s}$, respectively) of age and hindlimb propulsion at 10.5 $(0.226 \pm 0.016 \mathrm{~s}$ vs. $0.277 \pm 0.009 \mathrm{~s}$, respectively) and 12 $(0.234 \pm 0.009 \mathrm{~s}$ vs. $0.29 \pm 0.011 \mathrm{~s}$, respectively) months of
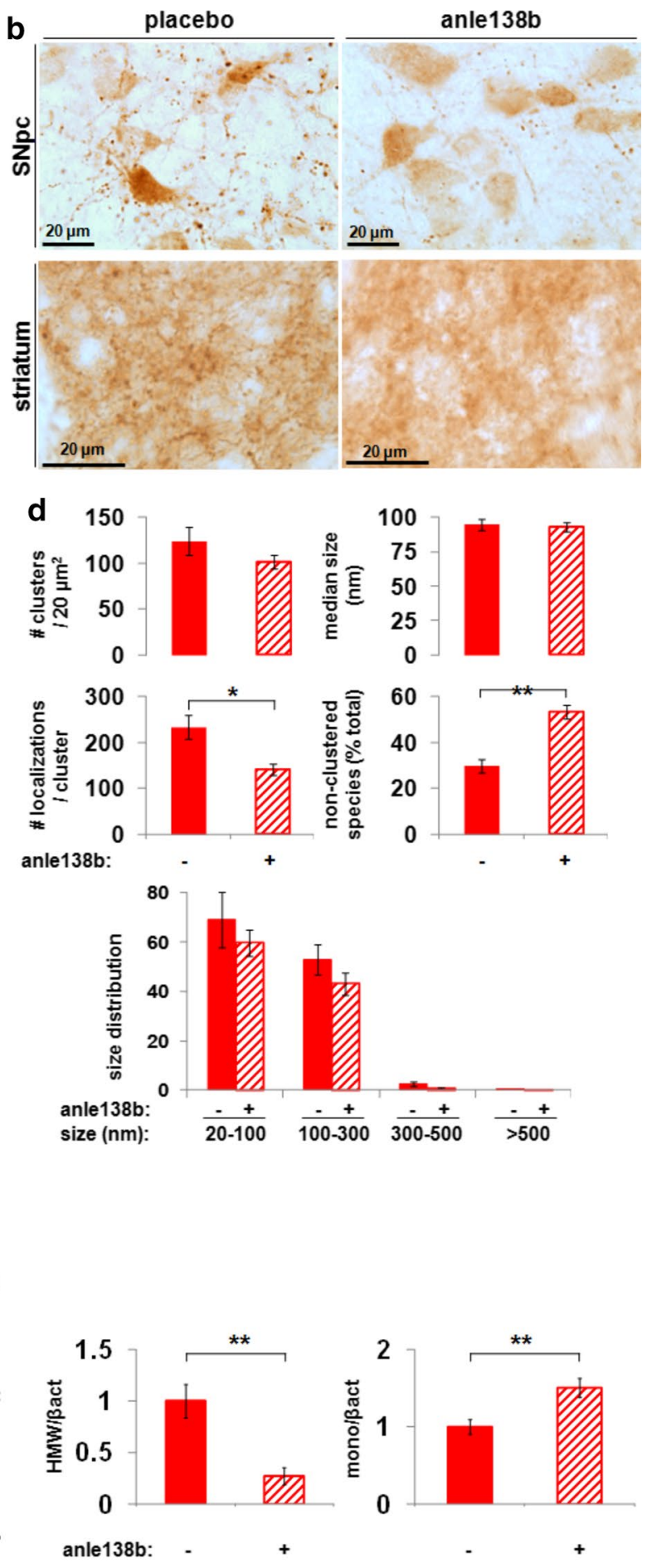

age was still decreased. However, we also observed statistically significant differences between anle138b-treated MI2 mice and anle138b-treated C57B1/6S mice in hindlimb stride length $(4.144 \pm 0.062 \mathrm{~cm}$ vs. $4.578 \pm 0.169 \mathrm{~cm}$, respectively) and stance duration $(0.313 \pm 0.006 \mathrm{~s}$ vs. $0.341 \pm 0.012 \mathrm{~s}$, respectively) at 12 months of age (Fig. 9). Taken together, 
४Fig. 7 Effect of anle138b on $\alpha$ Syn aggregation in MI2 mice. a Mice were treated with anle138b starting at 9 months and analyzed at 12 months of age. b Treatment with anle138b reduces the accumulation of 1-120 hoSyn in SNpc and striatum of MI2 mice as shown by Syn1 antibody immunostaining. c Representative $d$ STORM images of 1-120 hoSyn staining in the striatum of 12 month old MI2 mice treated with placebo (top panel) or anle138b (bottom panel). Large aggregates appear less dense and an increased number of smaller species are seen following anle138b treatment. d Quantification of $d$ STORM data revealed a significant decrease in the inner density of aggregates (\#localizations/cluster) and a significant increase in the percentage of non-clustered 1-120 h $\alpha$ Syn in anle138b-treated MI2 mice compared to placebo-treated littermates (see also Supplementary Fig. S8, Online Resource 1) (mean \pm SEM, $n=3$ mice; $t$ test, $\left.{ }^{*} p<0.05,{ }^{* *} p<0.01\right)$ while the number of clusters and their size did not change significantly. e Immunoblotting of striatal brain extracts from 12 month-old mice shows a decrease of $\alpha$ Syn high molecular weight (HMW) bands (arrowhead) and an increase of monomeric $\alpha$ Syn (arrow) in the striatum of anle138b-treated MI2 mice compared to MI2 placebo treated. 1-120 haSyn levels are also shown for 1.5, 9 and 12 month-old untreated MI2 mice. C57B1/6S (C57S) not expressing endogenous $\alpha$ Syn are shown as negative control. The star denotes non-specific bands recognized by Syn 1 antibody. Blot shows levels of $\alpha$ Syn before and after anle $138 \mathrm{~b}$ in 3 mice representative of 5-6 mice per group tested. Right panel-quantification of HMW 1-120 hoSyn and monomeric (mono) 1-120 haSyn after normalization to the levels of $\beta$-actin in all 5-6 mice tested (fold difference compared to placebo-treated mice \pm SEM, $n=5-6$ mice per group, $t$ test: ${ }^{* *} p<0.01$ )

these results show that targeting $\alpha$ Syn aggregation by anle $138 \mathrm{~b}$ rescues the DA release deficit, several aspects of the gait impairment and protects against cell loss, even when treatment was started when synaptic functional impairment was already present.

\section{Discussion}

In PD, $\alpha$ Syn aggregation is associated with a reduction of total striatal DA, a decrease in striatal DA release and nerve cell loss in the SNpc which, when reaching around $50 \%$, leads to overt motor impairment [5, 18]. Animal models recapitulating the progressing nature of $\alpha$ Syn pathology and DA dysfunction in PD are essential for testing new therapies. Here, we describe the MI2 mice, a new transgenic mouse model that expresses 1-120 hoSyn and shows a high similarity to PD pathological features with progressive loss of DA and a deficit in striatal DA release, $\alpha$ Syn aggregation in SNpc neurons and their striatal terminals, dopaminergic neuronal death in the SNpc, as well as motor deficits as initial fine alterations in gait pattern, followed by significant impairment in motor function as detected by rotarod test when cell death reaches around $50 \%$, similar to the human condition.

Previously we described another mouse line ( $\alpha$-Syn 120 mice) expressing the same $\alpha-S y n$ 1-120 transgene under the same TH promoter, that also showed progressive $\alpha$ Syn aggregation, SNARE protein redistribution and striatal DA dysfunction, however differently from the MI2 model these mice did not show DA neuron death in the SNpc and reduction of total DA in striatum occurred already at 3 months of age and was not progressive but remained constant [12, 35], when, in the MI2 mice reduction of total striatal DA occurred instead at 12 months of age. In another model expressing 1-119 $\alpha$ Syn in TH neurons DA neuronal death was not found [8]. Why our two $\alpha$ Syn 1-120 transgenic mouse lines differ is not clear but it could depend on the transgene insertion site in the mouse genome (chromosome 10 in MI2 and chromosome 3 in $\alpha$-Syn120 mice) or be related to the expression level of the transgene that although expressed under the same promoter appears lower in the MI2 mice compared to the $\alpha$-Syn 120 line. Further studies are needed to clarify the reasons for the differences between our two 1-120 $\alpha$ Syn transgenic lines.

Dopaminergic pathology in MI2 mice was associated with changes in 1-120 hoSyn protein distribution in SNpc and striatum accompanied by redistribution of SNARE proteins such as VAMP 2 (Supplementary Fig. S1, Online Resource 1) similar to what we have previously described in transgenic $\alpha$-Syn 120 mice and PD brains [12] and was also reported in DLB cases [19]. In SNpc of MI2 mice 1-120 hoSyn appeared in neuronal cell bodies as multiple small inclusions at 1.5 months of age, while large LB-like structures were present in numerous cells by 12 months of age. Some of these $\alpha$-Syn deposits were resistant to PK digestion and a small number of cells with $\alpha$-Syn aggregates contained also ubiquitin. Neuronal processes in the SNpc contained large dotted inclusions at 1.5 months of age, while in older mice processes were filled with dense aggregates, some of which exhibited PK resistance. This pattern indicates a progression in $\alpha$ Syn aggregation from small to large, less soluble structures, similar to human $\alpha$-synucleinopathies [21]. In the striatum of 1.5 month-old MI2 mice, 1-120 hoSyn deposits were small, but widely distributed in the neuropil, and in older animals they were more frequent and of larger size. Both, in SNpc and striatum, the $\alpha$ Syn aggregates became more insoluble with time, as shown by the appearance of stable HMW bands in immunoblots of MI2 mouse brain extracts, and increased amounts of $\alpha$ Syn in detergent-insoluble fractions in sequential extraction experiments. PK treatment appeared to fully digest the striatal $\alpha$ Syn aggregates while this was not the case for the aggregates present in the SNpc. However, it cannot be excluded that in the striatum some $\alpha$ Syn aggregates survive the PK treatment but could be too small to be detected by conventional microscopy.

To further characterize the progression of striatal synaptic 1-120 hoSyn aggregation, we used $d$ STORM, which allows detection of protein distribution at single molecule level and $20 \mathrm{~nm}$ resolution. Although $d$ STORM has been successfully employed to study synaptic proteins [2-4, 11, 34] we believe that this is the first time that $d$ STORM is used to analyze 

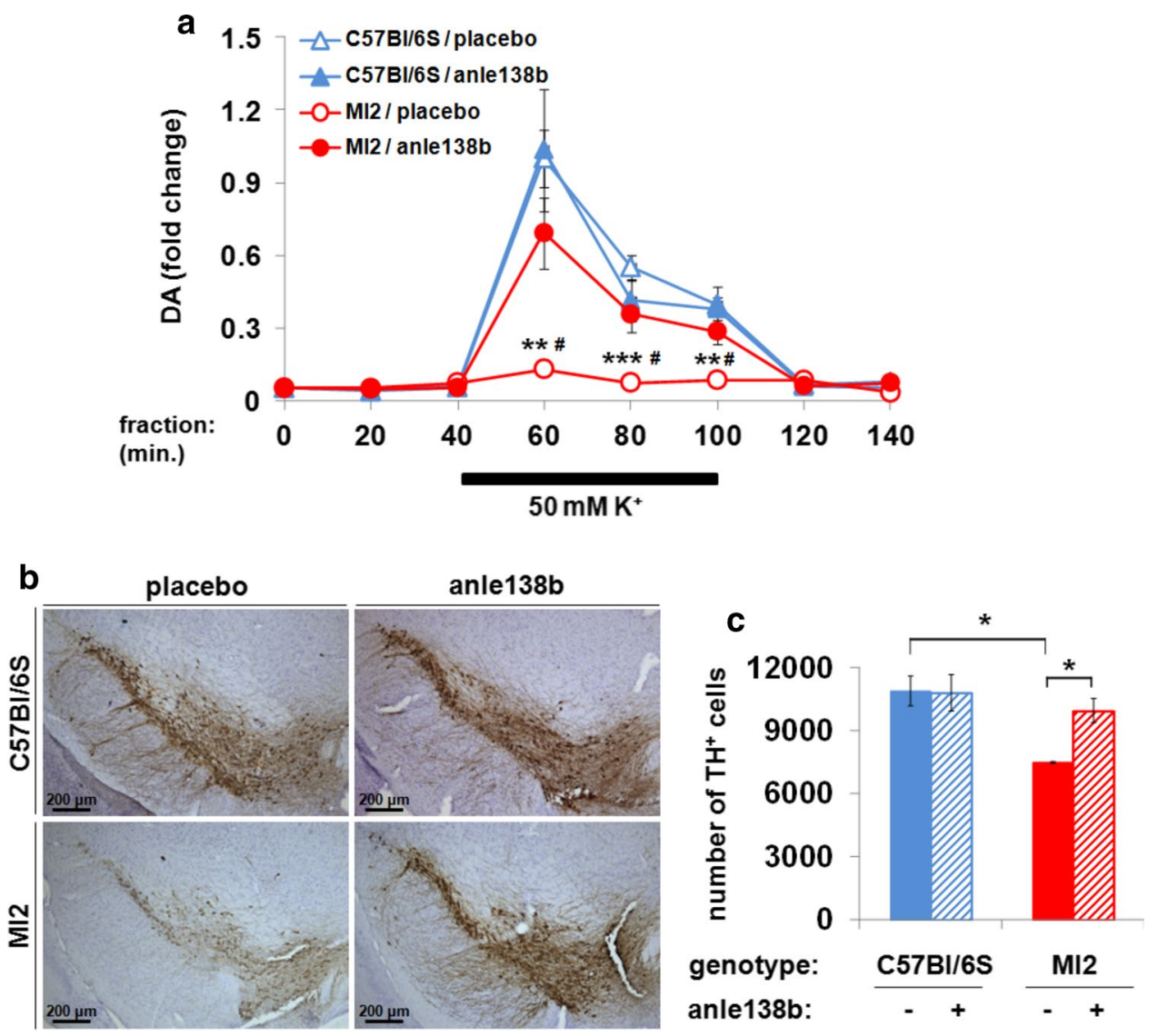

Fig. 8 Effect of anle138b on striatal DA release and SNpc DA neuron death in MI2 mice. a The effect of anle138b treatment on striatal DA release studied by in vivo microdialysis. Data are normalized to the value obtained in the $\mathrm{C} 57 \mathrm{Bl} / 6 \mathrm{~S} /$ placebo group at 60 min (mean \pm SEM, $n=5-6$ mice per group). Three-way mixed ANOVA identified an effect of genotype, sampling time, two-way genotype $\times$ sampling time interaction, and sampling time $\times$ genotype $\times$ treatment three-way interaction. Statistically significant differences in $\mathrm{K}^{+}$-induced DA release at 60,80 and $100 \mathrm{~min}$ were found between placebo-treated MI2 and C57B1/6S mice $(* * p<0.01$, $* * * p<0.001)$ and between placebo- and anle138b-treated MI2 mice $\left({ }^{\#} p<0.01\right)$ (multiple comparisons with Bonferroni correction). b

the progression and rescue of presynaptic $\alpha$ Syn pathology in vivo in brains of a PD mouse model. $d$ STORM confirmed a progressive aggregation of 1-120 hoSyn in the MI2 mouse striatum. Of all the parameters analyzed, cluster abundance and density (number of clusters per $20 \mu^{2}$ ) of 1-120 hoSyn aggregates appeared to be the factors best associated with the reduction in striatal DA release. The presence of $\alpha$ Syn aggregates has been recently demonstrated by some of us using a different super resolution microscopy method (DNA PAINT) in induced pluripotent stem cells (iPSCs) from a patient with a triplication of the SNCA gene [38], however, one of the unique findings of the present study is the differential age-dependent effect on aggregate size. We found that only 20-500 $\mathrm{nm}$ aggregates were increased with age rather
Preservation of TH immunoreactivity in SNpc of anle138b-treated MI2 mice compared with placebo-treated littermates. c Effect of anle138b treatment on nigral DA neuronal death determined using stereology (average number of nigral TH-positive cells \pm SEM, $n=3$ mice per group). A main effect of genotype was identified by two-way ANOVA. Anle138b treatment resulted in increased number of $\mathrm{TH}^{+}$ cells in MI2 mice compared to placebo-treated littermates $\left({ }^{*} p<0.05\right)$. Placebo-treated MI2 mice had less nigral $\mathrm{TH}^{+}$cells than placebotreated $\mathrm{C} 57 \mathrm{~B} 1 / 6 \mathrm{~S}$ mice $\left({ }^{*} p<0.05\right.$, multiple comparisons with Bonferroni correction) (see detailed statistical evaluation in Online Resource)

than aggregates larger than $500 \mathrm{~nm}$. Thus, as the small $\alpha$ Syn species $(<250 \mathrm{~nm})$ cannot be observed using conventional microscopy, establishing $d$ STORM as a novel approach for the analysis of $\alpha$ Syn aggregation in vivo in mouse models.

Striatal impairment of DA release was observed in MI2 animals before the measurable loss of DA cells in SNpc and DA innervation in the striatum (6 vs 12 months). This is consistent with our previously reported $\alpha$-Syn 120 mice, where a deficit in striatal DA release was present in the absence of DA neuron death in SNpc [12]. This observation supports the hypothesis that synapses play a central role in PD pathogenesis, being the primary site of dysfunction before loss of neurites and neurons occur, supporting the notion of retrograde degeneration $[6,10]$. 

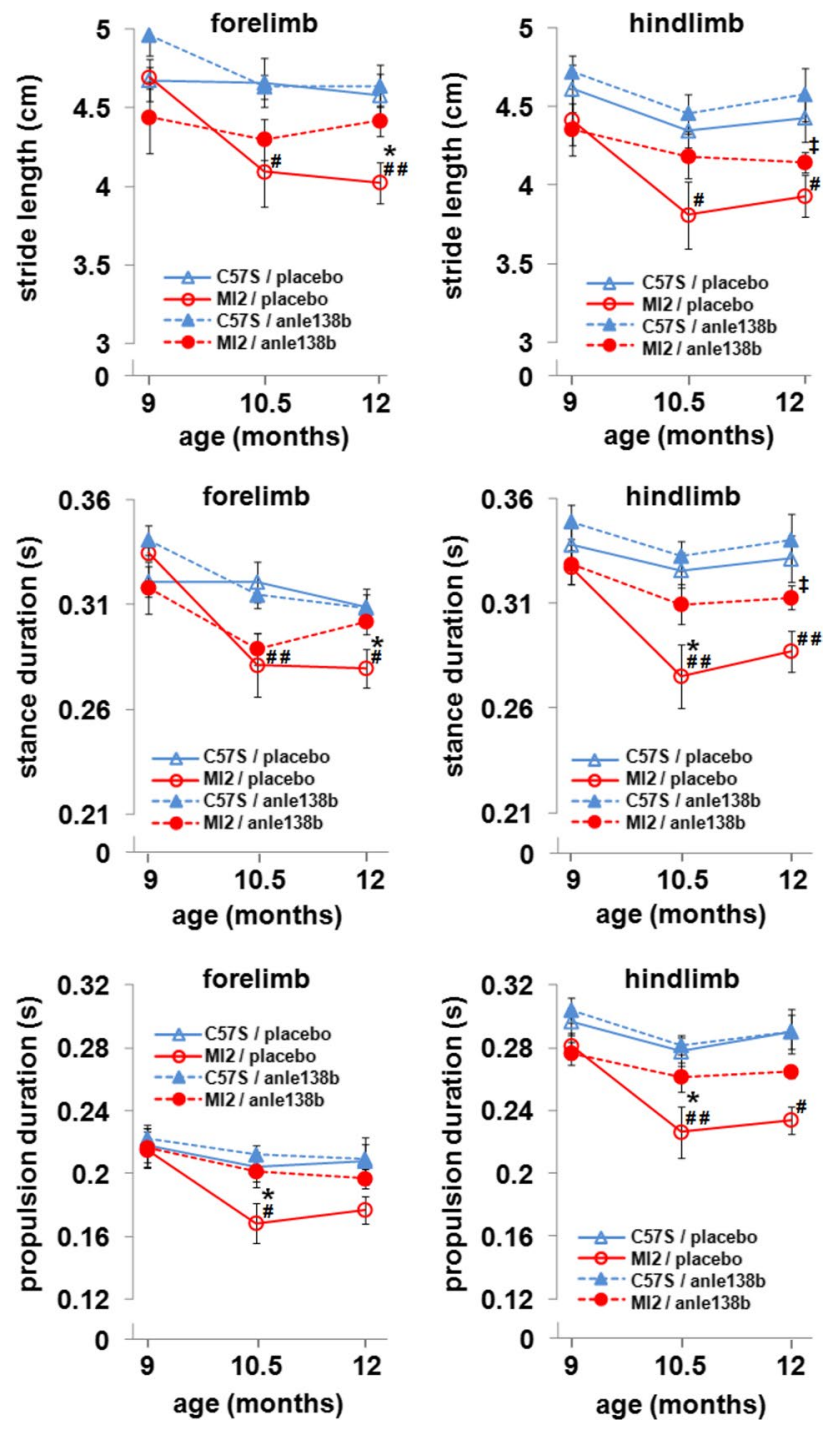

Fig. 9 Effect of anle138b on gait pattern in MI2 mice (see also Supplementary Fig. S9, Online Resource 1). Placebo- or anle138b-treated C57B1/6S and MI2 mice were analyzed using the DigiGait test. Treatment with anle $138 \mathrm{~b}$ rescued alterations in the duration of several of gait phases in MI2 mice (mean \pm SEM, $n=6-12$ mice per group; males and females). Three-way ANOVA revealed a main effect of age and a main effect of genotype on forelimb and hindlimb stride length, and stance and propulsion duration, a main effect of treatment on hindlimb stance and propulsion duration and a three-way age $\times$ genotype $\times$ treatment interaction for forelimb stance duration. At 10.5 months of age, statistically significant differences were found between placebo-treated C57B1/6S and MI2 mice in forelimb and hindlimb stride length, and in forelimb and hindlimb stance and propulsion duration $\left({ }^{\#} p<0.05,{ }^{\# \#} p<0.01\right)$, and between placebo- and anle138b-treated MI2 mice in hindlimb stance duration and in forelimb and hindlimb propulsion duration $\left({ }^{*} p<0.05\right)$. At 12 months of age, statistically significant differences were identified between placebotreated C57Bl/6S and MI2 mice in forelimb and hindlimb stride length and stance duration, and in hindlimb propulsion duration ${ }^{\#} p<0.05$, $\left.{ }^{\# \# p} p<0.01\right)$. At this age, a difference was found between anle138b-treated C57B1/6S and MI2 mice in hindlimb stride length and stance duration $\left({ }^{\ddagger} p<0.05\right)$ and between placebo- and anle138b-treated MI2 mice in forelimb stride length and stance duration $\left({ }^{*} p<0.05\right)$ (multiple comparisons with Bonferroni correction) (see detailed statistical evaluation in Online Resource)
Overt motor phenotype occurred in MI2 mice when around $50 \%$ of SNpc DA neurons were lost similar to human PD [5, 18], however subtle changes in gait pattern were found earlier. We observed decrease in the propulsion duration, resulting in reduced duration of stance, that, together with shorter swing phase, lead to decreased length of the stride. This phenotype can be compared with the walking alterations characteristic for PD patients, where shorter stride length is often observed and is believed to be related with slow, shuffling gait characteristic for the disease [22, 25]. Decrease in stride has also been reported before in other models of PD, including transgenic A53T mice [27], and pharmacological MPTP model [14]. We then used MI2 mice to test whether DA pathology might be rescued by targeting neuronal $\alpha$ Syn aggregation with anle138b, which has previously been shown to have beneficial effects by interacting specifically with structural epitopes of various protein aggregates and to rescue protein aggregation and related pathological features in models with $\alpha$ Syn, prion and tau aggregation [23, 36, 37]. Unlike the MI2 mice, the $\alpha$ Syn transgenic models used in previous anle138b studies did not present with consistent neuronal nigrostriatal $\alpha$ Syn aggregation or $\alpha$ Syn-related progressive dopaminergic impairment with motor impairment related in some cases more to spinal cord pathology [37] or $\alpha$ Syn pathology in oligodendrocytes [15]. Thus, our study is the first to investigate the effects of anle $138 \mathrm{~b}$ in a model with nigrostriatal neuronal DA alterations and neuronal $\alpha$ Syn aggregation as observed in PD. We show that a 3 month-treatment with anle138b (started at 9 months of age, when a deficit in striatal DA release but no significant neuronal loss is present) rescues DA dysfunction, prevents nerve cell death, and, to a large degree, protects from gait deficits. The reason why not all gait abnormalities are rescued by anle $138 \mathrm{~b}$ treatment remains to be investigated.

The results of our study are of particular significance because they show the presence of a time window, when DA function can still be rescued and neuronal death prevented despite the presence of $\alpha$ Syn-related synaptic alterations and subtle behavioral changes. Anle138b significantly changed $1-120 \mathrm{~h} \alpha$ Syn aggregation in the striatum and SNpc of MI2 mice, as observed by immunohistochemistry and immunoblotting. However, more information on its mechanism of action in vivo was obtained by $d$ STORM, which showed an increase in the percentage of small, non-clustered $\alpha$ Syn species in the striatum following anle $138 \mathrm{~b}$ administration. These species are likely to represent monomers and small assemblies of monomeric $\alpha$ Syn as determined by comparing their size to the size of recombinant hoSyn spread on a coverslip and immunostained as in the brain tissue and similarly examined with $d$ STORM. This analysis was an approximation since it compared the transgenic truncated $1-120$ protein with full-length recombinant $h \alpha$ Syn but this 
was done because the recombinant truncated protein has a greatly increased tendency to aggregate [7] making it difficult to determine the presence of bona fide monomeric species.

Moreover, after anle138b treatment, dense aggregates showed a significant reduction in inner density. This suggests that anle138b in vivo could interfere with aggregation by preventing the formation of new aggregates and/or the recruitment of non-aggregated $1-120 \mathrm{~h} \alpha \mathrm{Syn}$ into existing aggregates possibly destabilizing them, thus leading to a reduction of their inner density, resulting in both cases in an increase in small, non-clustered $\alpha$ Syn species in the environment. Whether the anle138b effect is related to one or both of these mechanisms could be only established by monitoring aggregation of hoSyn with $d$ STORM in anle138b-treated MI2 mice over time.

It remains to be clarified, how anle $138 \mathrm{~b}$ produced its beneficial effect, whether the effect is related to the reduced toxicity of less dense aggregates or to the increase in small $\alpha$ Syn species, which could be physiologically active and restore DA release. It will be interesting to see if removal of small $\alpha$ Syn species by inducing their degradation can potentiate the effects of anle138b by further reducing possible aggregation or reduce its effect, as it could be expected if the small $\alpha$ Syn species are functional.

In summary, we report here a transgenic mouse model that recapitulates the progressive $\alpha$ Syn aggregation and DA neuron dysfunction and death observed in human PD. Striatal synaptic DA dysfunction preceded SNpc DA neuronal death and loss of striatal TH terminals, supporting synaptic dysfunction as being an early pathogenic factor in PD. Anle138b restored DA function, rescued several gait abnormalities and prevented nerve cell loss by disrupting $\alpha$ Syn aggregate formation as shown for the first time by using $d$ STORM. Overall, this study indicates that MI2 mice are a suitable model to test mechanism-based therapies for $\alpha$-synucleinopathies, and $d$ STORM is a useful technique for studying structural/morphological changes related to aggregate toxicity. This work also indicates that there is a window of time when it is possible to prevent DA neuronal death, even when striatal DA release is already impaired.

Acknowledgements We acknowledge the help of Ms Emma Carlson in the in vivo microdialysis experiments and preparation of tissue for immunohistochemistry. We thank Drs Aviva Tolkovsky and Michel Goedert for useful discussions and comments on the manuscript. We are also grateful to the personnel in the Bioscience facilities, in particular, Ms Debbie Drage who has helped us generate and maintain the MI2 mouse line in that due to several changes of bioscience facilities the mice had to be re-derived and a new colony started several times over the years. This work was supported over the years by Parkinson's UK, the Cure PD Trust, the MJ Fox Foundation, UK Medical Research Council, Alzheimer's Research UK, TEVA, Neuroscience Network of Excellence (NNE), Israel Science Foundation grants 2546/16 and 953/16, the Max Planck Society, and the Deutsche
Forschungsgemeinschaft (DFG, German Research Foundation) under Germany's Excellence Strategy_EXC 2067/1-390729940.

Author contributions MGS planned and supervised the study and provided funding; AG, CG provided anle138b; UA supervised the $d$ STORM and contributed funding for it; MW performed the immunoblots, immunohistochemistry, microdialysis, stereological and behavioral experiments, maintained mouse colonies, and supervised the anle138b treatment; DB-O performed $d$ STORM imaging and analysis; LC supervised treatment with anle138b, and assisted in microdialysis experiments; OA was involved in the initial characterization of the MI2 mice, and supervised the anle $138 \mathrm{~b}$ treatment; MI produced the transgene used to generate the MI2 line; JX and JWD performed the dopamine measurements; SR and AL were involved in developing the anle $138 \mathrm{~b}$ compound; MW and MGS wrote the manuscript with contributions from UA, DB-O and CG. The manuscript was revised by all authors.

\section{Compliance with ethical standards}

Conflict of interest A. G. and C. G. are co-founders of MODAG. A. L. is partly employed by MODAG.

Research involving animals All applicable international, national, and/ or institutional guidelines for the care and use of animals were followed. This research has been performed under the Animals (Scientific Procedures) Act 1986 Amendment Regulations 2012 following ethical review by the University of Cambridge Animal Welfare and Ethical Review Body (AWERB), under project license no. 70/8383.

Open Access This article is distributed under the terms of the Creative Commons Attribution 4.0 International License (http://creativeco mmons.org/licenses/by/4.0/), which permits unrestricted use, distribution, and reproduction in any medium, provided you give appropriate credit to the original author(s) and the source, provide a link to the Creative Commons license, and indicate if changes were made.

\section{References}

1. Baba M, Nakajo S, Tu PH, Tomita T, Nakaya K, Lee VM et al (1998) Aggregation of alpha-synuclein in Lewy bodies of sporadic Parkinson's disease and dementia with Lewy bodies. Am J Pathol 152:879-884

2. Bar-On D, Nachliel E, Gutman M, Ashery U (2011) Dynamic conformational changes in munc18 prevent syntaxin binding. PLoS Comput Biol 7:e1001097

3. Bar-On D, Wolter S, van de Linde S, Heilemann M, Nudelman G, Nachliel E et al (2012) Super-resolution imaging reveals the internal architecture of nano-sized syntaxin clusters. J Biol Chem 287:27158-27167

4. Bielopolski N, Lam AD, Bar-On D, Sauer M, Stuenkel EL, Ashery U (2014) Differential interaction of tomosyn with syntaxin and SNAP25 depends on domains in the WD40 beta-propeller core and determines its inhibitory activity. J Biol Chem 289:17087-17099

5. Brooks DJ, Salmon EP, Mathias CJ, Quinn N, Leenders KL, Bannister R et al (1990) The relationship between locomotor disability, autonomic dysfunction, and the integrity of the striatal dopaminergic system in patients with multiple system atrophy, pure autonomic failure, and Parkinson's disease, studied with PET. Brain 113:1539-1552 
6. Calo L, Wegrzynowicz M, Santivanez-Perez J, Grazia Spillantini M (2016) Synaptic failure and alpha-synuclein. Mov Disord 31:169-177

7. Crowther RA, Jakes R, Spillantini MG, Goedert M (1998) Synthetic filaments assembled from C-terminally truncated alphasynuclein. FEBS Lett 436:309-312

8. Daher JP, Ying M, Banerjee R, McDonald RS, Hahn MD, Yang L et al (2009) Conditional transgenic mice expressing C-terminally truncated human alpha-synuclein (alphaSyn119) exhibit reduced striatal dopamine without loss of nigrostriatal pathway dopaminergic neurons. Mol Neurodegener 4:34

9. Deacon RM (2013) Measuring motor coordination in mice. J Vis Exp 75:2609

10. Decressac M, Mattsson B, Lundblad M, Weikop P, Bjorklund A (2012) Progressive neurodegenerative and behavioural changes induced by AAV-mediated overexpression of alpha-synuclein in midbrain dopamine neurons. Neurobiol Dis 45:939-953

11. Fulterer A, Andlauer TFM, Ender A, Maglione M, Eyring K, Woitkuhn $\mathrm{J}$ et al (2018) Active zone scaffold protein ratios tune functional diversity across brain synapses. Cell Rep 23:1259-1274

12. Garcia-Reitbock P, Anichtchik O, Bellucci A, Iovino M, Ballini C, Fineberg E et al (2010) SNARE protein redistribution and synaptic failure in a transgenic mouse model of Parkinson's disease. Brain 133:2032-2044

13. Garcia-Reitboeck P, Anichtchik O, Dalley JW, Ninkina N, Tofaris GK, Buchman VL et al (2013) Endogenous alpha-synuclein influences the number of dopaminergic neurons in mouse substantia nigra. Exp Neurol 248:541-545

14. Goldberg NR, Hampton T, McCue S, Kale A, Meshul CK (2011) Profiling changes in gait dynamics resulting from progressive 1-methyl-4-phenyl-1,2,3,6-tetrahydropyridine-induced nigrostriatal lesioning. J Neurosci Res 89:1698-1706

15. Heras-Garvin A, Weckbecker D, Ryazanov S, Leonov A, Griesinger C, Giese A et al (2018) Anle138b modulates alpha-synuclein oligomerization and prevents motor decline and neurodegeneration in a mouse model of multiple system atrophy. Mov Disord $34: 255-263$

16. Hindle JV (2010) Ageing, neurodegeneration and Parkinson's disease. Age Ageing 39:156-161

17. Jellinger KA, Lantos PL (2010) Papp-Lantos inclusions and the pathogenesis of multiple system atrophy: an update. Acta Neuropathol 119:657-667

18. Kordower JH, Olanow CW, Dodiya HB, Chu Y, Beach TG, Adler $\mathrm{CH}$ et al (2013) Disease duration and the integrity of the nigrostriatal system in Parkinson's disease. Brain 136:2419-2431

19. Kramer ML, Schulz-Schaeffer WJ (2007) Presynaptic alphasynuclein aggregates, not Lewy bodies, cause neurodegeneration in dementia with Lewy bodies. J Neurosci 27:1405-1410

20. Kudwa AE, Menalled LB, Oakeshott S, Murphy C, Mushlin R, Fitzpatrick J et al (2013) Increased body weight of the BAC HD transgenic mouse model of Huntington's disease accounts for some but not all of the observed HD-like motor deficits. PLoS Curr 5:e49838

21. Kuusisto E, Parkkinen L, Alafuzoff I (2003) Morphogenesis of Lewy bodies: dissimilar incorporation of alpha-synuclein, ubiquitin, and p62. J Neuropathol Exp Neurol 62:1241-1253

22. Lewis GN, Byblow WD, Walt SE (2000) Stride length regulation in Parkinson's disease: the use of extrinsic, visual cues. Brain 123:2077-2090

23. Martinez Hernandez A, Urbanke H, Gillman AL, Lee J, Ryazanov S, Agbemenyah HY et al (2018) The diphenylpyrazol compound anle138b blocks A-beta channels and rescues disease phenotypes in a mouse model for amyloid pathology. EMBO Mol Med 10:32-47

24. McFadyen MP, Kusek G, Bolivar VJ, Flaherty L (2003) Differences among eight inbred strains of mice in motor ability and motor learning on a rotorod. Genes Brain Behav 2:214-219

25. Morris ME, Iansek R, Matyas TA, Summers JJ (1996) Stride length regulation in Parkinson's disease. Normalization strategies and underlying mechanisms. Brain 119:551-568

26. Mouton PR, Gokhale AM, Ward NL, West MJ (2002) Stereological length estimation using spherical probes. J Microsc 206:54-64

27. Paumier KL, Sukoff Rizzo SJ, Berger Z, Chen Y, Gonzales C, Kaftan E et al (2013) Behavioral characterization of A53T mice reveals early and late stage deficits related to Parkinson's disease. PLoS One 8:e70274

28. Paxinos G, Franklin KBJ (2004) The mouse brain in stereotaxic coordinates. Elsevier Academic Press, Amsterdam

29. Perrin RJ, Payton JE, Barnett DH, Wraight CL, Woods WS, Ye L et al (2003) Epitope mapping and specificity of the anti-alphasynuclein monoclonal antibody Syn-1 in mouse brain and cultured cell lines. Neurosci Lett 349:133-135

30. Sigal YM, Zhou R, Zhuang X (2018) Visualizing and discovering cellular structures with super-resolution microscopy. Science 361:880-887

31. Specht CG, Schoepfer R (2001) Deletion of the alpha-synuclein locus in a subpopulation of C57BL/6J inbred mice. BMC Neurosci 2:11

32. Spillantini MG, Crowther RA, Jakes R, Hasegawa M, Goedert M (1998) alpha-Synuclein in filamentous inclusions of Lewy bodies from Parkinson's disease and dementia with lewy bodies. Proc Natl Acad Sci USA 95:6469-6473

33. Spillantini MG, Schmidt ML, Lee VM, Trojanowski JQ, Jakes R, Goedert M (1997) Alpha-synuclein in Lewy bodies. Nature 388:839-840

34. Tang AH, Chen H, Li TP, Metzbower SR, MacGillavry HD, Blanpied TA (2016) A trans-synaptic nanocolumn aligns neurotransmitter release to receptors. Nature 536:210-214

35. Tofaris GK, Garcia Reitbock P, Humby T, Lambourne SL, O'Connell M, Ghetti B et al (2006) Pathological changes in dopaminergic nerve cells of the substantia nigra and olfactory bulb in mice transgenic for truncated human alpha-synuclein(1-120): implications for Lewy body disorders. J Neurosci 26:3942-3950

36. Wagner J, Krauss S, Shi S, Ryazanov S, Steffen J, Miklitz C et al (2015) Reducing tau aggregates with anle138b delays disease progression in a mouse model of tauopathies. Acta Neuropathol 130:619-631

37. Wagner J, Ryazanov S, Leonov A, Levin J, Shi S, Schmidt F et al (2013) Anle138b: a novel oligomer modulator for disease-modifying therapy of neurodegenerative diseases such as prion and Parkinson's disease. Acta Neuropathol 125:795-813

38. Whiten DR, Zuo Y, Calo L, Choi ML, De S, Flagmeier P et al (2018) Nanoscopic characterisation of individual endogenous protein aggregates in human neuronal cells. ChemBioChem 19:2033-2038

Publisher's Note Springer Nature remains neutral with regard to jurisdictional claims in published maps and institutional affiliations. 